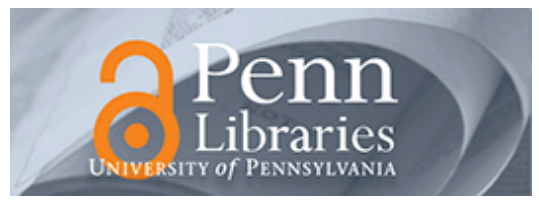

Studies in Visual Communication

1984

\title{
Why Dance Films Do Not Look Right: A Study in the Nature of the Documentary of Movement as Visual Communication
}

Virginia Loring Brooks

\section{Recommended Citation}

Brooks, V. L. (1984). Why Dance Films Do Not Look Right: A Study in the Nature of the Documentary of Movement as Visual Communication. 10 (2), 44-67. Retrieved from https://repository.upenn.edu/svc/ vol10/iss $2 / 5$ 
Why Dance Films Do Not Look Right: A Study in the Nature of the Documentary of Movement as Visual Communication 


\section{Why Dance Films Do Not Look Right: A Study in the Nature of the Documentary of Movement as Visual Communication}

\section{Virginia Loring Brooks}

\section{Sources of Error in the Perception of Dance on Film}

The actual performance of a dance is an event that occurs in a particular space over real time; John Martin (1965) terms it a "formal entity." How we perceive such a performance when it is presented on a large movie screen or a small television screen is subject to a number of variables that provide possible sources of perceptual error. These variables reflect the interaction between the characteristics of the display on the screen and those of the real world (that is, the rhythm and the composition of the choreog raphy and of the performance)

Just about everyone who cares at all agrees that dance in film does not look right. In the theater the audience experiences the inherent rhythm and the composition of the choreography. The audience viewing the event on the screen, however, experiences the rhythm imposed by the shots selected by the editor and the composition selected by the cameraman through framing. Both of these factors are under the control of the director, who ideally should work in collaboration with the choreographer; unfortunately, this is not always the case.

What happens to the composition and rhythm we experience in the theater when it is transposed to the screen, which has its own composition and rhythm?

The composition of the motion picture frame is, in fact, a major source of perceptual error because it affects the way the space of the performance area will be perceived. The way the performance area is perceived, in turn, determines how the depth and magnitude of the movement that occurs in that field will

Virginia Brooks is a freelance filmmaker and videorecordist in the field of dance. She is currently teaching in the Film Department of Brooklyn College, C.U.N.Y., where she is deputy chairman for production. From 1978 to September 1981 she was head of the Jerome Robbins Film Archive of the Dance Collection of The New York Public Library at Lincoln Center, during which time she wrote her doctoral dissertation, "The Art and Craft of Filming Dance as Documentary," at Columbia University. Dr. Brooks is the author and coauthor of numerous research papers and chapters on perception and motion pictures, and the author of reviews and reports for Dance Magazine, Film News, and the Dance Research Journal. She is the director and distributor of two films, School of American Ballet and Isadora Duncan, Technique and Choreography, as well as over sixty films and videotapes of choreographic record. appear. This topic can be divided into four separate categories, the first two being determined by the dancers' relationship to the screen's frame and the second two being determined by their relationship within the screen's frame:

1. The dancers' relationship to the total performance area when the screen's frame equals the whole performing area

2. The dancers' relationship to the screen's frame when only a selected area of the total performance space fills the frame

3. The dancers' relationship to each other within the screen's frame

4. The dancers in terms of their own body space within the screen's frame

Errors also arise in the crucial area of perceived time and rhythm. This topic includes, of course, the objectively measured rhythm of the music (or other accompaniment) and the relationship of the choreography to it. In addition, we must consider the performance, i.e., the dancers' phrasing as it is seen to relate to the musical measure and as it affects how effort and energy level are perceived when the dance is translated to the screen. Perhaps less obviously, this topic must also include the subjective or per ceived rhythm of the music as it relates to the choreography and as the choreography is affected by cuts, camera movement, and camera placement. Other factors to be investigated in the perception of time and rhythm are the effects of repetition versus change and the rate of change versus the complexity and comprehensibility of the display. There has been a long history of theory and research in psychology on the question of how apparent time is affected by the perceived amount of material with which it is filled (Ornstein 1969). The results of most of these experiments have been inconclusive; the adequate measures have not yet been isolated, and anecdotal evidence suggests that research is still needed.

These are the two general areas in which the perception of staged and filmed dance are likely to be different. This article attempts to do two things: to identify the sources of error in perceiving the composition and the rhythm of the choreography and performance of dance as they occur in existing films and tapes of dance performance, and to formulate the principles that should govern the relationship between dance and film in those films that are intended to document how a live dance performance is experienced. These analyses and generalizations are undertaken first for the problems of composition and then for problems of rhythm. This enterprise is a valuable one not only for those concerned with the specific task of improving the quality of films of dance but more widely for those interested in the visual communication of all movement. 


\section{Composition: Perception of the Space of the Field and the Perception of Movement in That Space}

\section{The Entire Performance Area Revealed by the Stationary Camera}

What are the factors that will influence the way in which we perceive the choreography as performed by the dancers when the screen displays the whole stage area, i.e., when the camera need not move to include the whole scene? The performance presented in the real, three-dimensional world differs from the performance viewed on a flat screen, and we must begin by considering these differences in a general way.

Of course, the screen is flat or slightly curved. In order to represent a real performance in any way, the image must provide information about the spatial layout of the represented performance, that is, it must provide depth cues. Depth cues are the characteristic patterns that are produced when the light from a three-dimensional layout is projected on a two-dimensional surface. Many of the static depth cues are familiar from their use in still pictures: linear perspective, occlusion or interposition (the interruption of a far object by a near one), size perspective (the image of a far object being smaller than that of the same object nearby), texture-density gradient (the image of a surface composed of pebbles, tiles, grass, etc., showing progressive decrease in size from near to far), and height in the field or relation to the horizon. Perspective was analyzed formally by Brunelleschi in 1413 (Kemp 1978); the first three static depth cues were prescribed by Leonardo da Vinci in 1457 (Richter 1970); the fourth category was analyzed by Gibson (1950) and the fifth by Sedgwick (1980). These cues provide the viewer with information that the image on the flat surface is related to (that is, that it represents) an arrangement of objects at different distances in space (Haber 1979). These static cues also apply to film and video when a stationary camera is directed at a static layout, within which motion may, of course, occur.

In addition, there are motion depth cues that are produced when there is relative motion between the viewer and the layout: motion perspective, in which the near objects move faster on the screen than far ones; kinetic occlusion, in which the leading edge of an object progressively hides more of the background and in which the trailing edge progressively discloses more of the background; and the opticalflow pattern, in which all of the texture elements and other discernible contours in the field of view move away (with faster velocities for nearer objects) from the image of the point toward which the viewer or camera is moving and at which it is directed. This last cue will be important in the next section when the camera's, and therefore the audience's, point of view will be moving.

Now that we have considered the representation of depth in a general way, we must look at five specific sets of factors that will be important in performances presented on the screen: the background, the floor, the aspect of ratio of film as it compares to the proscenium arch, the distribution of objects in foreground and midground, and the lighting.

The background. The background is one of the most important factors in the perception of the space of the field and of the depth and dimension of the movement when one is looking at a display that includes the whole performance area.

A homogeneous background, such as a uniformly lit cyclorama or a dark backdrop that produces the effect of dancers moving in limbo, makes easier the task of editing shots together and matching them seamlessly (Hochberg and Brooks 1978). At the same time, the eye is given no reference point against which to judge the size of the movement of the dancers (Duncker 1938, Vorkapich 1972).

When a dancer or a group of dancers is moving against a homogeneous background, a cut (or change in direction of the angle of the camera) is disorienting to the viewer, even when the shot includes the whole performance area. This is so because the viewer has no information as to whether the dancers have moved or the camera has moved. The viewer of a live performance has no such problem, because he "knows" whenever he has changed his direction of gaze. The viewer of a live performance must turn his head and make eye movements over a large area. The film viewer looks at a much smaller area, depending on how far he sits from the display, and there are no proprioceptive cues from the muscles of his body that relate the views on the screen to their loci in space. This fact may be used purposely in filming in order to produce a particular "floating" effect, in which the dancers seem almost to be in freefall (this is done in the San Francisco Ballet film Tealia and in Birgit Cullberg's "Red Wine in Green Glasses"), but it may also destroy the viewer's grasp of the dance space and the choreographic structure.

If there are several dancers on stage, some of them may act as reference points for the others, provided that they are stationary; this may be seen in "Trinity" (City Center Joffrey Ballet, Dance in America series) in which the active dancers' movements are perceived in relation to a set of static dancers who are posed at the sides and back of the set, which is a plain cyclorama. 
When the background is part of a realistic set or contains a pattern or some distinguishing landmarks (Hochberg, Brooks and Roule, 1977; Hochberg and Gellman 1977), the situation is quite different. Now the dancers move against the visual framework that is provided by the pattern; they are seen passing before it, and the value and extent of their movements are preserved, as is not the case with the homogeneous background. ${ }^{2}$

The detail of the background is far more critical in tape and film than it is in the theater, because in the former case the detail is actually in the same plane with everything else that the viewer is watching. That is, both the background and the dancers are in the plane of the screen. This is a very important point: None of the depth cues that are used to create apparent depth change the fact that the image on the film screen or video monitor is two-dimensional and that all the components of the scene are in fact at a single distance from the viewer. In the theater, of course, an actual physical distance extends between the dancers and the scenery, and the viewer's eyes can converge and focus on the plane of the dancers, or on that of the background, but not on both. Unless extremely selective focus is used, therefore, the detail in the background will have much more visual impact and consequence on the screen than it does in the theater.
The floor. Almost equal in importance to the background, particularly when it is homogeneous, is the floor as a factor in perceiving depth.

Because the screen is flat, the dancers' movement toward or away from the camera may be perceived less as motion in depth than as the change in size that their images on the screen undergo as their distance from the camera changes. When the background is homogeneous, therefore, and there are no salient depth cues, the floor itself is a very important factor to be considered.

A pattern or grid on the floor will provide a cue for depth because it offers a gradient of perspective on the screen: that is, the horizontal and vertical lines of the grid will be farther apart the closer they are to the front of the stage set and will converge toward the back. This contributes to the perception that, as they travel on the vertical lines, the dancers are going back into space, rather than just appearing to become smaller. This phenomenon is apparent in both "Allegro Brillante" and in "Tschaikovsky Pas de Deux" ("Choreography by Balanchine, Part IV," Dance in America series).

An irregular pattern of any sort gives more of a cue to direction than does a homogeneous and featureless field, either on the background or on the floor, and it provides some information about depth (by providing on the screen a gradient of the texture's density [Gibson 1950]), but a nonrepetitive pattern does not carry the sense of depth that is given by a perspective pattern. ${ }^{3}$

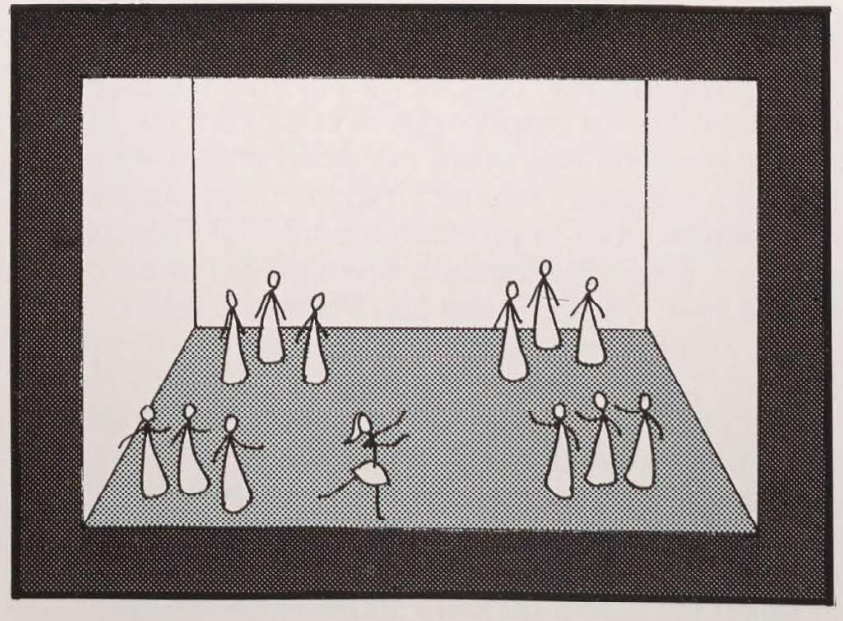

Figure 1a The proscenium arch and the stage filled with dancers. The spectator in the theater will be aware of the black frame (the darkened theater) surrounding the arch only if he turns his head or if he sits far away.

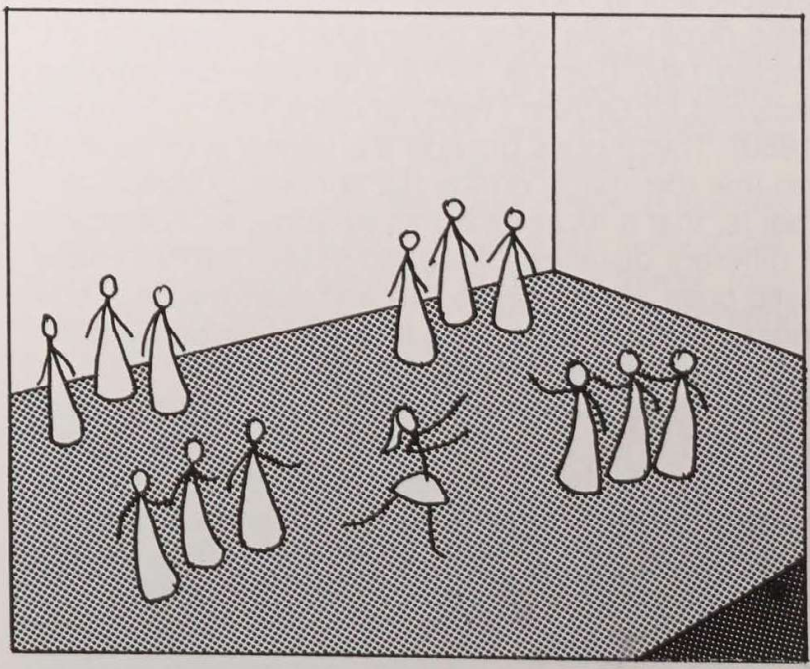

Figure 1b The standard Academy ratio format of film, 1.33 (width) to 1 (height), here imposed on the scene from straight on. If the entire width of the performing area is preserved, there will be a black unlit area below the dancers and a large area of unused lit headroom above them. 
The ratio of film compared with the proscenium arch. Another major factor that alters the visual impact of the dancers' movements on the screen is the amount and distribution of unlit space on that screen. For example, if the performance is taking place on a proscenium stage (Figure 1a), both the standard Academy ratio of the film frame (i.e., the proportion between the width and height of a projected picture, 1.33:1) and the cut-off of the television screen are such that the top third and the bottom third of the frame will be empty when the stage is shot head-on and the wings are at the side of the frame. The action will then be displayed only within the limits of the center third of the screen and will be "oppressed" or minimized by the large percentage of dark area (Figure 1b).

The extreme long shots from the top of the house in the Live from Lincoln Center series and many sequences shot in the theater for The Turning Point exemplify this problem. If the stage is filmed slightly from one side, the discrepancy will be lessened, but the area on that side of the stage will be distorted (Figure 1c). In the studio, the width of the performance area will be not so large, and even at the widest prospect the edge of the performance area will be defined by the edge of the frame, as will the top and bottom edges. The field filling the frame will be completely lit (Figure 1d). This can be seen in the final section of "The Four Temperaments" ("Choreography by Balanchine, Part I," Dance in America series). I contend that this sequence seems to have even more impact (by which I mean the ability to convey to the audience the energy of the choreography and its relationship to the music) than does either a film of the performance on the proscenium stage or the actual performance. In the first case, the field is filled and fully lit; in the case of the film, the field is not fully lit; and in the case of the performance, the field is not filled. This hypothesis has no research to support it, nor is it clear as yet how we could measure "impact" experimentally, but the importance of a frame has been discussed in general terms by others (Arnheim 1960, Gombrich 1972) and research efforts in this direction are probably worthwhile.

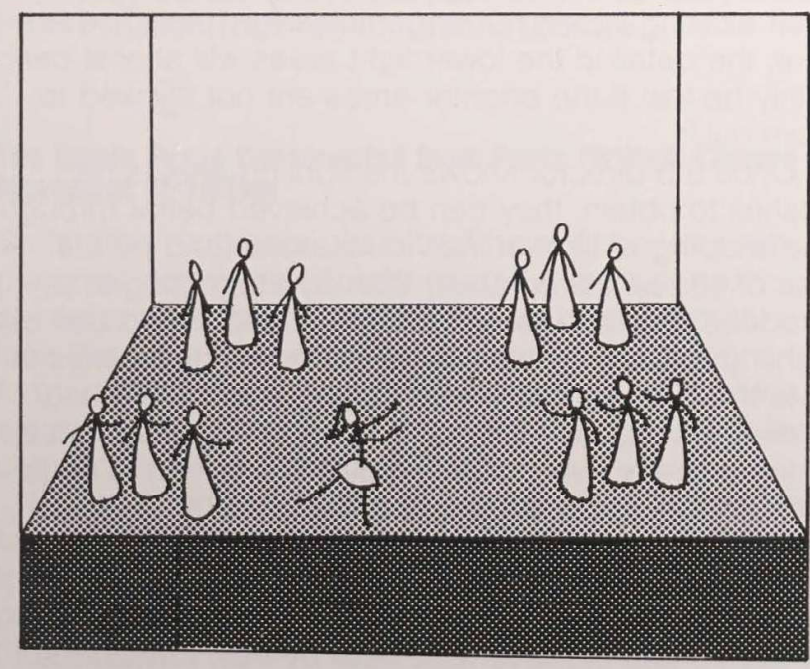

Figure 1c The standard Academy ratio imposed on the scene from slightly to one side. The sense of center is distorted, but more area is lit because the width requirements are reduced.

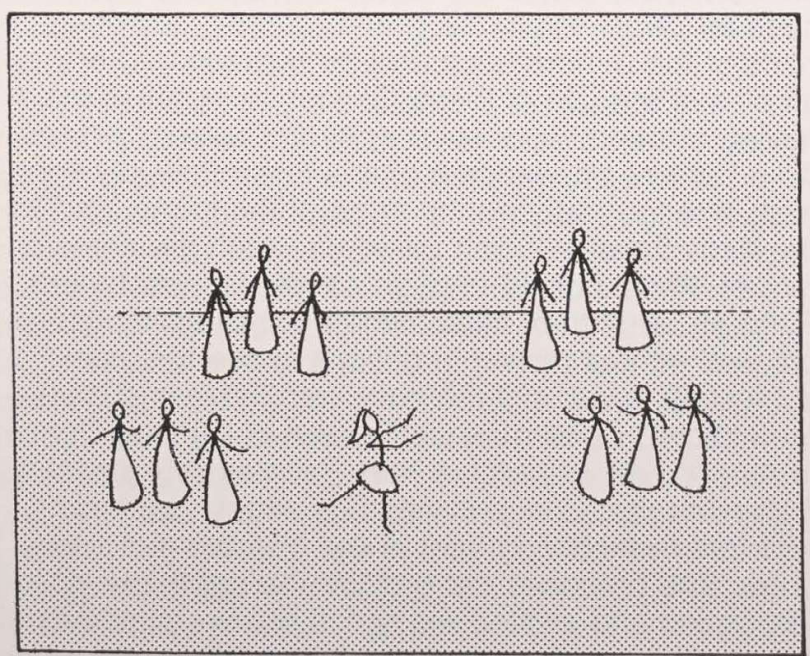

Figure 1d Fully lit framing of the entire performance area achieved in a studio. 


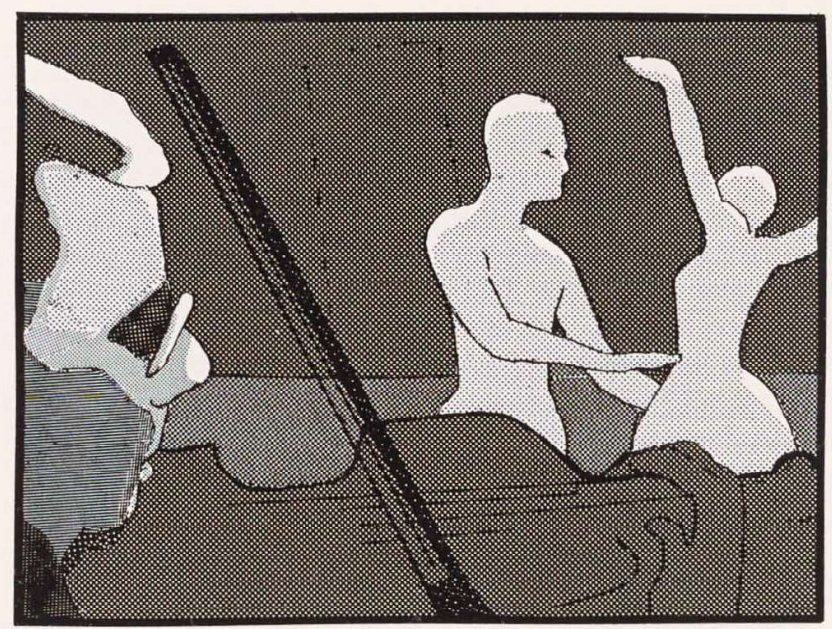

Figure 2 Duo Concertant (Balanchine, New York City Ballet, RM Productions). Dancers seen behind the musician. Depth is achieved at the expense of the comprehension of the choreography.
Distribution of objects, foreground and background. The visible field in which the performance occurs is sometimes restricted in other ways, even in the studio situation described above. In order to af fect the perception of depth and the orientation of the whole performance space, people or objects are placed in the foreground and midground of that space. Depending on how these elements are distributed and how they are lit, they may provide good cues for depth within the scene, or they may obscure parts of the performance and be distracting. Early examples of the use of these devices to increase the feeling of depth were seen in Birgit Cullberg's "Miss Julie," where nondancing characters in low-key light were arranged around the edge of the frame, and in the Kirov Ballet's "Swan Lake," in which the camera moves, while looking through candle flames, to find Rothbart. In both of these films a sense of depth was created without making it difficult to see the main subject. This was not the case with the less successful "Holberg Suite" (Dance Theatre of Harlem, Dance in America series) and in "Les Patineurs" (American Ballet Theatre, Dance in America series); both films had tree branches in front of the dancers in some shots. The branches did provide the depth cue of interposition and elicited the sense of several planes of space, but they also made the shots feel cluttered. A classically bad example is seen in Duo Concertant (RM Productions film of the Balanchine ballet) where, in some scenes, the dancers are observed from behind the violinist (Figure 2), an angle and position from which one would hardly choose to watch the dance, even though it did provide some depth cues through occlusion.

The lighting. Finally, how the scene is lit is an extremely important factor in how the performance will be perceived on the screen. The general light level must be high enough to satisfy the technical requirements of the film or tape, and a great many filmed or taped performances seem to take this as their sole criterion. But other criteria are based on aesthetic requirements as well as the need for legibility: lighting that is designed to produce shadows on the floor will enhance the feeling of depth; lighting from the sides provides a means of modeling the bodies and helps to separate them from the background; and the use of different levels of light on figures dancing in different planes (background, midground, and foreground) increases both the feeling of depth and the legibility or comprehensibility of the image.

Lights may also be used to isolate one area from another, as in regular stage lighting. Efforts to recreate stage lighting, however, or attempts to film or tape in a performance situation with unaugmented stage lighting often fail because attention is not paid to the continuously varying exposure levels and the overall light level needed to obtain a good image on film or videotape. Indeed, the range from light to dark that is often found in the theatrical lighting of a single scene poses different problems for videotape and for film. For the former, a three to one ratio of the lightest to darkest area is ideal; a somewhat higher ratio is possible with film, but a much higher base level of light is required, and this is a very serious limiting factor. ${ }^{4}$ In taping and filming stage productions, with their existing lighting arrangements (or "plots"), therefore, the detail in the lower light areas will almost certainly be lost if the brighter areas are not allowed to burn out. 5

Once the director knows the lighting effects he wishes to obtain, they can be achieved better through the shaping of light in individual areas (and by the use of sequential contrast) than by attempting to reproduce an entire range within a scene. Good use of lighting in this way was achieved by Ralph Holmes in "Martha Graham Dance Company" and "Sue's Leg/ Remembering the Thirties" (Dance in America series).

In summary, when the frame of the screen is equivalent to the whole stage or performance area (i.e., when the camera doesn't have to move because everything is in the frame), the following. factors are potential sources of error in the perception of space, of depth, and of movement (and by this, I mean the impact of the movement that is the heart of the dance, as well as the perceived magnitude and direction of the movement): 
1. If the background is homogeneous, it may be difficult or impossible for the viewer to keep track of the orientations and locations of the dancers through the course of a sequence of cuts from different angles, since the eye is given no reference point against which to judge the size and direction of the dancers' movements.

2. If the background is a patterned cyclorama or a realistic set, its details may compete with the dancers in significance, because the dancers and background are both being presented in the same visual plane, that is, the plane of the television or motion picture screen.

3. A homogeneous, featureless floor will give no cues to depth or direction of movement and may result in disconcerting changes in perceived size.

4. The dimensions of the proscenium stage when filmed straight from the front do not match standard film ratio; I believe that the resultant unlit space reduces the impact of the performance. Filming from the side lessens the discrepancy but distorts the viewer's sense of center.

5. Elements placed in the foreground or midground of the set to achieve a sense of depth may make the frame appear cluttered and actually obscure parts of the performance to be recorded.

6. Finally, the level of lighting may be too low for adequate recording, or the design of the lighting may be too flat to provide the separation and shadows that are needed as depth cues or it may be too uneven to provide the consistent illumination required for ideal exposure.

\section{The Whole Scene Constructed from Parts through Camera Movement or Editing}

So far, the problems described have been very simple ones-almost artificially simple. We must next consider what the choreography and the dancers look like when one can see only a part of the performance area at a time and when other parts of the stage can be seen only by moving the camera or by cutting from one shot to another.

Background is found to be an even more important factor when the camera moves continuously to keep a traveling dancer in view and the screen frame encompasses only a section of the performance area. When the dancer is moving against a homogeneous background and the camera is also moving, the viewer has no way to determine how much of the motion on the screen is due to the camera's motion and how much is due to the progress of the dancer. No sense of the size and direction of the movement will be pro- vided by the homogeneous background (Duncker 1938, Vorkapich 1972). In a realistic set or in one with markings on the backdrop or with patterned lighting on the cyclorama, these elements will move on the screen in the opposite direction, behind the dancer, who is being kept in frame by the panning camera. this opposing motion will increase the impact of the dancer's ongoing movement. ${ }^{6}$

A ubiquitous aspect of the background is the horizon line, or wall-to-floor line. That line moves down and up in the frame as the camera follows a dancer moving up and down. If it is deemphasized by the use of a cove (a curved molding) and special lighting, as is often done in a television studio, the wall-tofloor line that moves up and down in the frame as shots change angle will be less noticeable. This is not without cost, however, since a noticeable horizon line does provide a clearer frame of reference for the height of the dancers' jumps and an indication of the distance that the dancers move backward and forward in the set. The movement of the line may be minimized, where it is objectionable, if the dancers are kept in frame by camera movement rather than by cuts, so that the abrupt transitions that cause jumps of the horizon are eliminated even if the line itself remains clearly defined.

Another set of factors that will be very important when the space of the performance area is assembled through camera movement or cuts is the size and angle of the frame. The size and angle of the frame affect the perceived depth of the represented space and, therefore, affect as well the apparent speed and size of the dancers' movements. These relationships are worth examining in some detail because they must affect all representations of motion and not only the films of dance.

When the camera is stationary, the size of the frame determines the distance available for the dancers to cross; it also defines the true value of the movement on the screen itself. In a closeup, a large movement may fill the screen, and whereas the step of which the movement is a part may be identifiable from the detail available in the very near shot, the compositional value of the step will not be the same as it would be if the entire movement were visible: Even a small movement will cover a larger visual angle of space than it would in the theater and, therefore, will have a different value in the composition as a whole. The general fact is that the closeup presents a much narrower field of attention than would be applied normally to watching dance. As such, it may prove valuable before the dance begins or during an early pose to provide an opportunity to identify with the dancers. Research on this point would be very useful. 


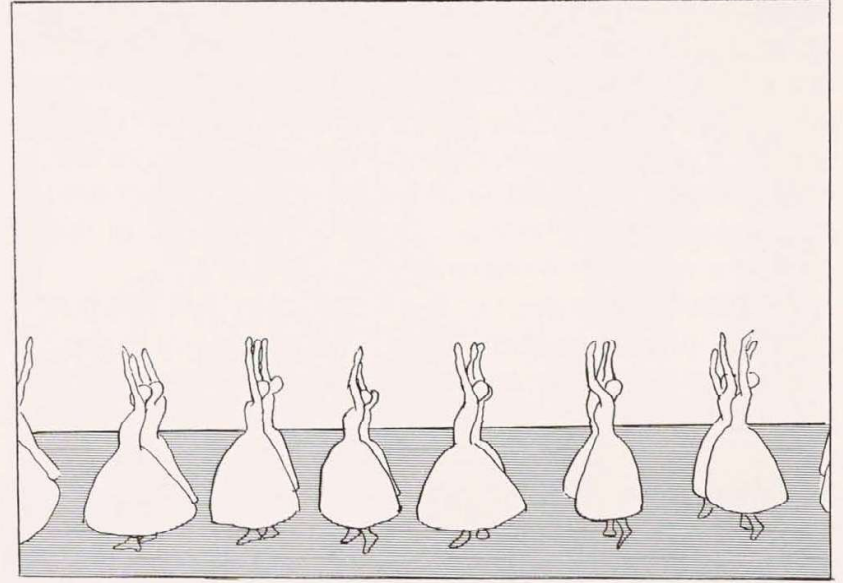

Figure 3a Les Sylphides (Fokine, American Ballet Theater, Live from Lincoln Center). Center shot of a formation of dancers from just above eye level. Dancers in front occlude most of the dancers in the second row.

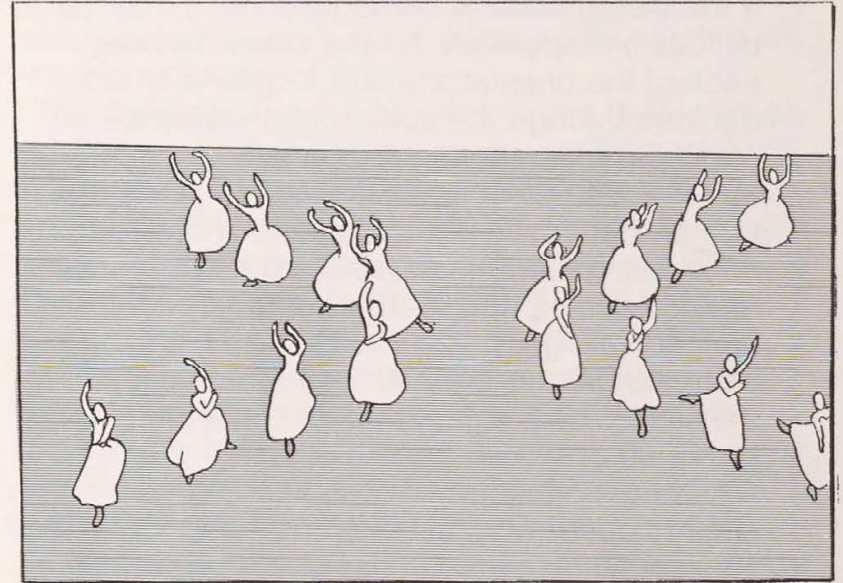

Figure 3b Center shot of same formation of dancers from a high angle showing the $X$ pattern formed by the two lines of dancers but losing the front to back interactions.
A medium shot will include slightly more of the performance space, but it is still the case that only part of a dancer will be shown. The medium shot is also a narrower focus of attention than is usually directed to dance, particularly since it does not afford the viewer the choice of changing his view, as his peripheral vision of the stage normally does

A medium long shot can include a full-length dancer with room for the arms extended to the sides or overhead. As a stationary camera shot, it can serve for turns in place, tight partnering, port de bras, mime sequences, and the like. In these cases, the movement perceived will be relatively true to life. When the dancer begins to travel, however, the camera will have to move fast in order to keep the dancer in the frame. A patterned background will then move correspondingly fast in the opposite direction, amplifying the movement and, perhaps, changing its relationship to the music, as we will discuss later. In addition, in a medium long shot there will be little opportunity to maintain orientation in the frame similar to that in the whole performance space.

In a long shot, several dancers may be included: for instance, a principal couple and two soloists on either side in the horizontal plane. In addition, the shot would include whatever number of dancers from the pattern of the choreography fit into the inverted triangle of the camera's space behind the front line of
Figures $4 \mathbf{a}, \mathbf{b}$, c Changing the focal length of the lens but maintaining the same distance from the camera to the subject simply changes the magnification of that subject on the screen while preserving the same perspective.

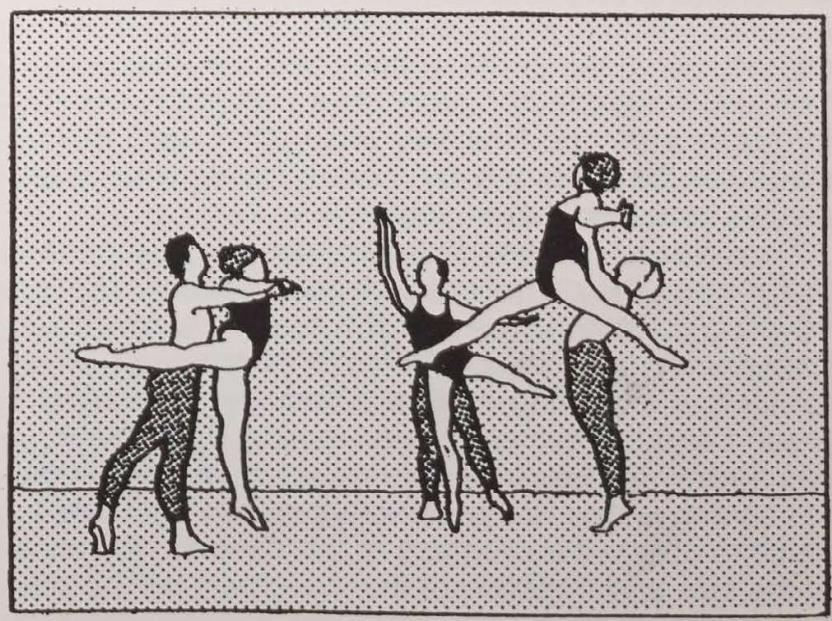




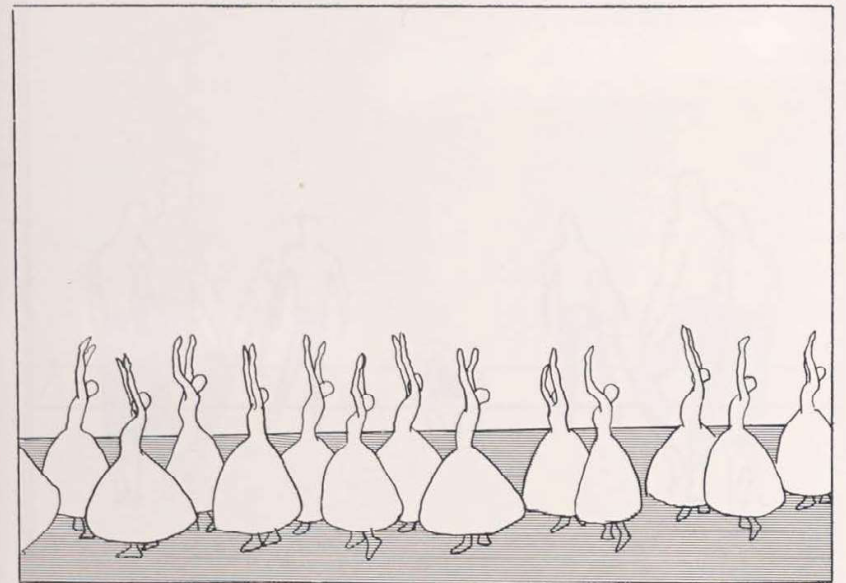

Figure 3c Same formation shot from slightly to one side, just above eye level, showing front to back interactions but losing the actual pattern.

dancers. The larger frame permits a wider range of dance movement in proportion to camera movement; the value of the movement may appear close to that in performance or it may be slightly diminished. As the frame gets larger, the angle from which the steps are being shot seems to become more critical, in terms of how these movements will be interpreted or read.

The very long shot and the extreme long shot will include the entire performance space; no camera movement is then required to keep the dancers in frame. In these cases, however, the size of the dancers' image and, correspondingly, the value of each of their movements are greatly decreased, because the distance that they displace on the screen is very small. In 1931, J. F. Brown reported on laboratory experiments showing that the perceived velocity of an object was proportional to the size of a stationary framework imposed around it. Despite this prediction, it seems that the apparent reduction in tempo experienced in the extreme long shot indicates a breakdown of motion constancy (the fact that things seem to move at the speed they are really moving regardless of their distance from the viewer) when the image size is this small.

In addition to the choice of field size, which is es tablished by the distance from the subject and focal length of the lens used, the director must consider the continuum of possible angles from which to shoot, ranging from low through eye level to high and from side to side through the center. The changes in height are important primarily for the shifting of the horizon line and for the amount of floor that is revealed. These changes determine whether the pattern of choreography will be seen against the floor or whether the dancers will be seen moving in front of each other and/or the background. This situation compares with that of a member of the audience whose angle of vision depends on whether he is sitting in the orchestra or the balcony. The center shot approximates the axis most preferred in the theater and the one on which choreography for the proscenium is based (Figure 3a). For the film or television screen, however, the center shot is not always the best choice for seeing either the overall pattern of the choreography or for making legible the individual steps. Unless the camera is high (Figure 3b), a shot taken slightly from the left or the right of center will be more appropriate if there is a formation consisting of more than one row of dancers (Figure 3c).
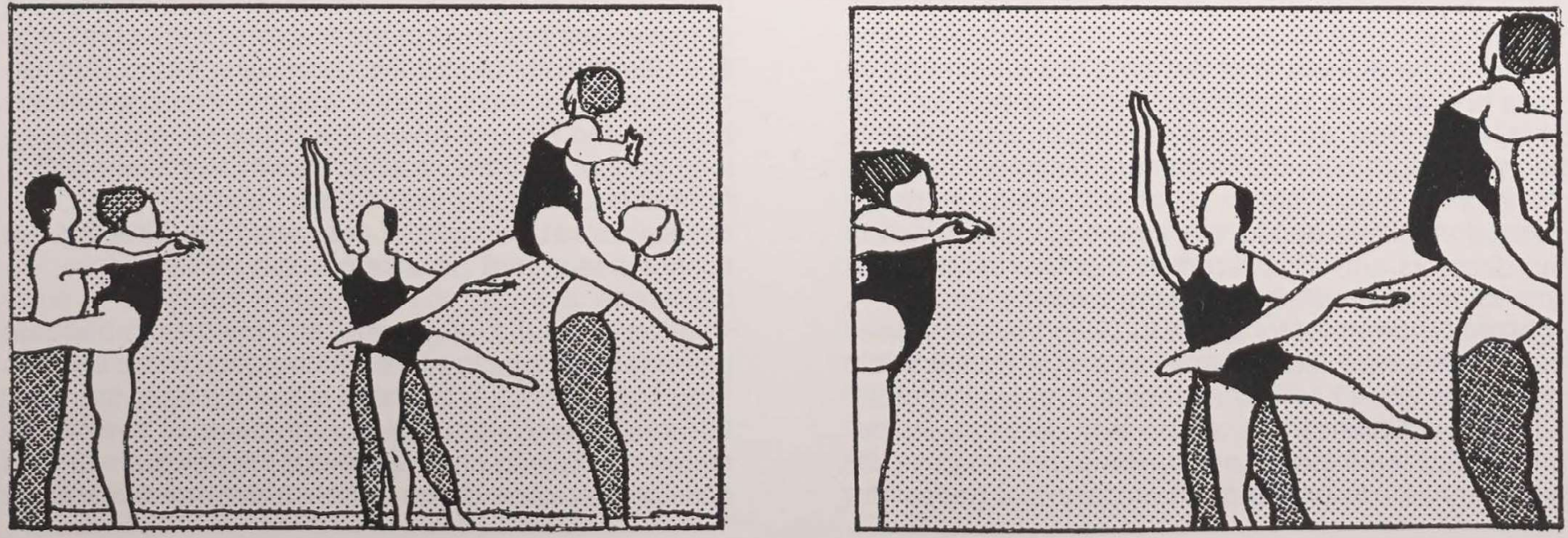


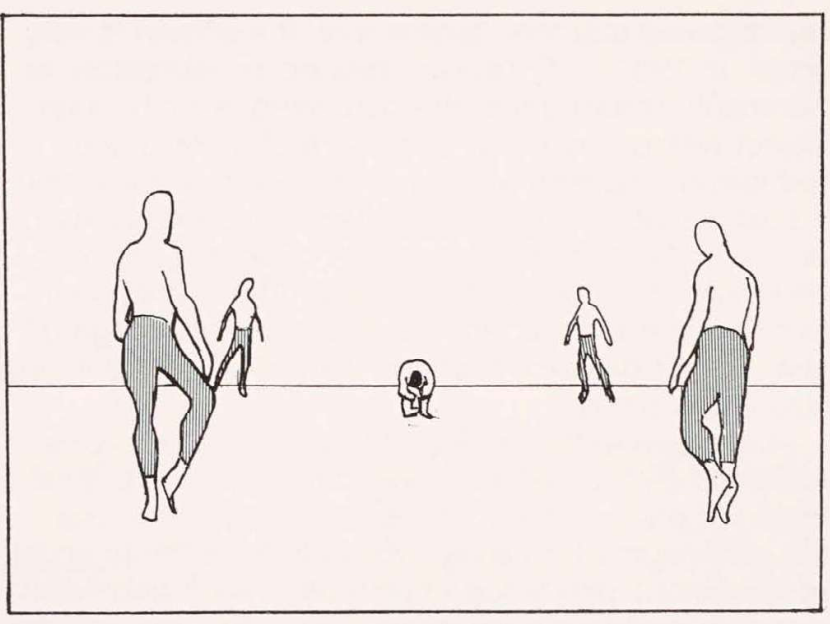

Figure 5a Four Temperaments (Balanchine, New York City Ballet, Dance in America). Wide-angle lens with the camera fairly close.

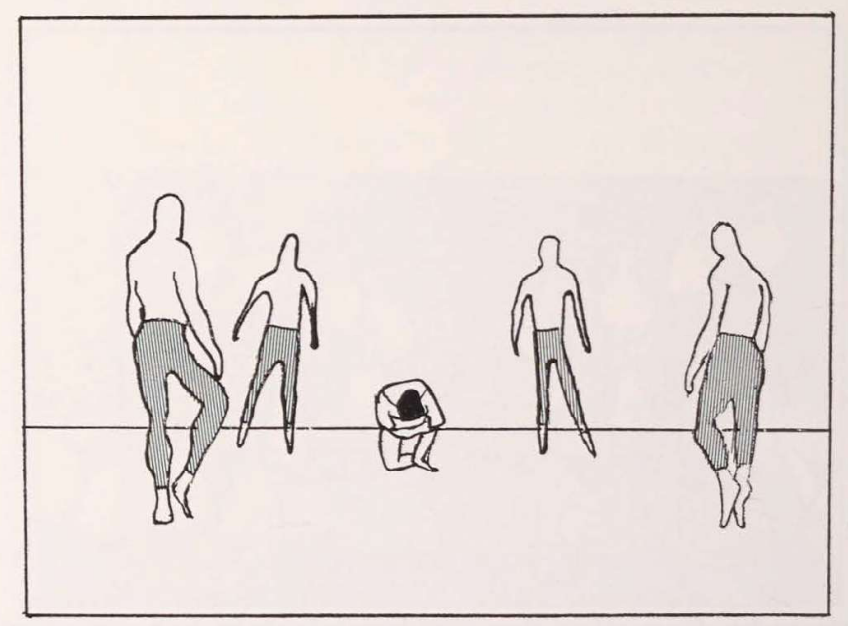

Figure 5h Telephoto lens with the camera farther away. The image size is nearly the same but the perspective has changed.
In all of the previous section we have noted that the size of the field and the angle from which that field is viewed will determine the graphic content of the shot, but the relationship that is most critical in the perception of movement filmed in depth is the one between the distance the camera is placed from the performing area and the focal length of the lens that is needed to obtain the desired field size from that distance (Figures $4 \mathrm{a}, \mathrm{b}, \mathrm{c}$ )

The camera distance determines the relative proportions of near objects (or dancers) to far objects, maintaining the size of the main subject using different focal length lenses as shown in Figures $5 \mathrm{a}$ and $\mathrm{b}$.

The focal length of the lens determines the amount of magnification applied to the proportions obtained at that distance, as in Figures $6 a$ and $b$. The perceived distance between the dancers results from the interaction between the proportions and the magnification.

Moving the camera in or out on a dolly, track, or crane changes the proportions of the dancers to each other; in contrast, zooming in or out (i.e., changing the focal length but not the camera distance) merely changes the magnification but retains the same proportions. All these factors are known to cameramen and directors and are utilized by them in narrative films to create a variety of effects; for instance, the wide-angle, deep-focus space in Long Voyage Home and the telephoto, compressed space of Benjamin's race to the church in The Graduate. The feeling of separation of planes and of opened-out space, in the former, and the futile race that makes no progress, in the latter, were intended to create physical bases for emotional states. The actual values of the movements were not really the subject of either film. In a film dance performance, however, movement is precisely the subject. Cutting from one lens to another, moving the camera, zooming, and changing the size of the field all distort the value of the movement (Hochberg in press). In dance, only the choreographer should be entitled to alter the movement: the director of the film or tape must be prepared to consult with the choreographer about any changes that may result through the use of specific camera techniques.

The size and angle of the frame, discussed above, should also be chosen with regard for the feeling intended by the choreographer. A stage densely crowded with dancers may not be perceived as crowded in a long shot that does in fact include them all but in which they fill only one-third of the frame (the top and bottom thirds would be black or empty of movement because of the standard aspect ratio). The decision should be the choreographer's. The choreographer must choose whether the shot should be long and high, to reveal the pattern (Figure $3 \mathrm{~b}$ ); long and at eye level, so that the whole pattern with front to back interactions is seen (Figure $3 c$ ); or closer, so that only part of the pattern and interactions are seen and a sense of the quantity is captured.

In the case of a single dancer, how much space is needed to convey the feeling intended by the choreographer? A full figure shot will emphasize the actual steps being performed or the detail of a mime sequence. A long shot will reveal the figure's aloneness on the stage or the dancer's movement through 


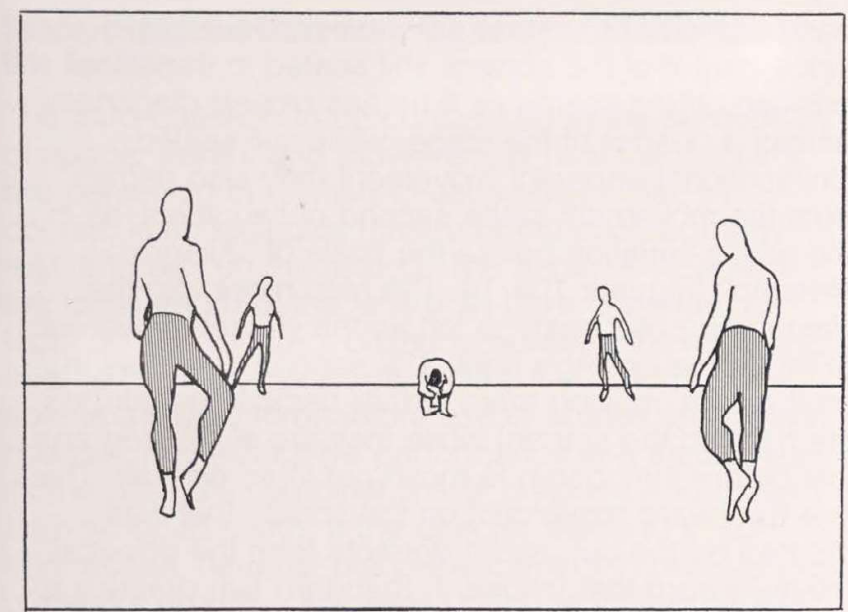

Figure 6a Wide-angle lens with the camera at the same distance as in Figure $5 \mathrm{a}$.

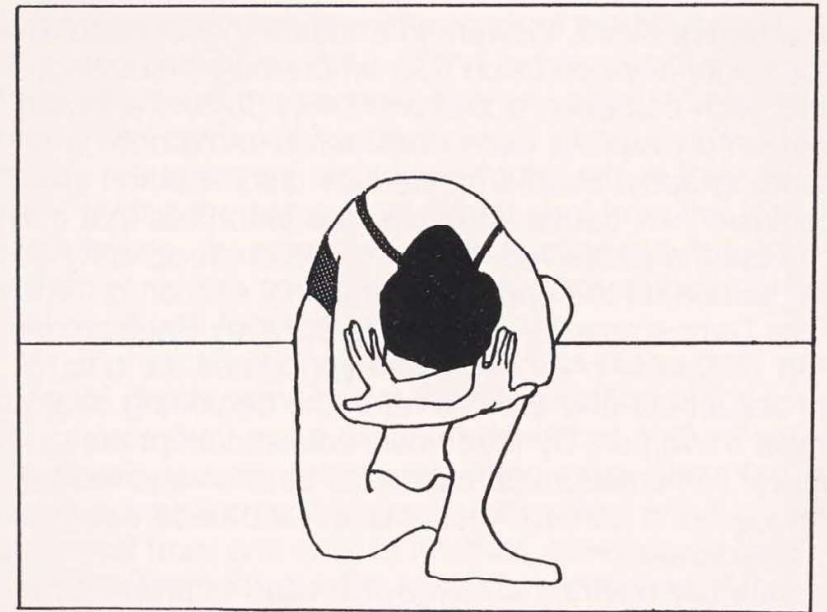

Figure 6b Telephoto lens with the camera at that same distance. The image has been magnified and includes a much smaller portion of the performance area. the whole space. The choreographer's intent must be the main determining factor in selecting the shots; but in order to preserve the relationship between the dancer's body and the steps, and between the steps and the choreography, no less than a medium long shot of one complete full figure, with no hand or foot missing, should be on the screen most of the time. The amount of detail needed to convey both the content and the feeling of the choreography will vary with the complexity of the dance, the pace of the dance, and the number of dancers on the stage.

The movement of the scene across the screen, whether it is obtained through the discontinuous transition of cutting from one shot to another or through the continuous transition of camera movement, must be thought out very carefully in relation to the choreography and to how the latter will be perceived when the camera moves. The director must be sure that no conflicting vectors will be produced, that enhancement or amplification of movement is appropriate, and that the choreography remains comprehensible despite the moves.

The movement of the frame should not create a vector that conflicts with the choreographed movement; that is, the frame should not move in a way that will unintentionally diminish or magnify the screen value of the stage movement. For example, a choreographed movement that is supposed to travel on the diagonal from upstage left to downstage right should not be shot after a cut is made to a shot from a camera located downstage right. It probably should not be shot from downstage left either, unless there were some other compositional reason for doing so, such as something going on upstage right. The scene should be shot from near the center to allow for the sense of the dancer crossing the stage on the diagonal. If the camera must move out or pan in order to keep the dancer in frame, it should not move, zoom out, or pan at a rate faster than the dance movement itself.

The camera movement may, in fact, enhance the choreographed movement by amplifying it, as when the camera pulls out at the same rate that two dancers are separating and, therefore, increases the distance between them on the screen available for them to move apart within. Conversely, if the dancers are coming together, pulling the frame in at the same rate not only increases the size of their images on the screen, it decreases the distance that separates them (Figures 7a, b, c, d). The camera may also magnify the choreographed movement by moving sympathetically, swinging from side to side as the movement swings. In the pas de deux from the balcony scene of "Romeo and Juliet" with Fonteyn and Nureyev (Figures $8 \mathrm{a}, \mathrm{b})$ or in the ballet sequence from "Limelight," in which the camera swings from side to side with Eglevsky's leaps, the sense of movement is amplified and elicits an especially pronounced feeling of participation or empathy from the viewer.

When the frame is changed by means of a discontinuous transition or cut, the orientation and direction of the perceived movement must be preserved either through the use of landmarks in the background or by the presence of other recognizable dancers on the stage. Clear and continuous comprehensibility are particularly important in reproducing the experience 
of a dance event. Viewers in a theater never need to cope with any confusion over where they are sitting, and such confusion about one's viewpoint is a factor that simply has not been considered in the original choreography. Even a momentary disorientation or confusion will cause an unplanned emphasis that may change the perceived rhythm of the choreography on the screen. In the opening of the final section of "The Four Temperaments" ("Choreography by Balanchine, Part I," Dance in America series) (Figures 9a, b, c, d), an abrupt cut and a dissolve on the beginning of a travel movement by the female soloist disrupt the viewer's orientation: at least a full beat is required for the viewer to achieve reorientation and reidentification of the dancers.

Carefully planned cuts may be used to prepare for a better-angle shot of a particular movement, but, as with camera movements, thought must always be given to the succeeding shot. The actual location of the figure or figures on the screen at the end of the first shot must be considered when planning the shot to follow, or unintentional and undesired apparent movement on the part of that stationary dancer (or an element of the scene) may result.

One reason that such unintentional apparent movement may be distracting is that it may represent movements that are physically impossible. Such an impossible movement occurs in the opening of "Elegy" ("Choreography by Balanchine, Part IV," Dance in America series). In the first shot, the man is seated on the floor downstage right, that is, in the lower-left- hand portion of the screen. In the second shot, he is in the center of the screen, still seated in the same position, and it seems as if he has moved diagonally, across a quarter of the stage, while still seated. Unintentional apparent movement may also detract from the movement in the second of two shots, as in the men's variation before the finale of "Allegro Brillante" (Figures 10a, b): The men move into the frame and pose upstage left as the women move out of the frame upstage right. The second shot cuts in on the men, moving them farther back stage left (to the right on the screen) while they are still posed and just before they begin to move out to screen left. This is a backward movement on the screen that was created by the cut, and it detracts from the physical move forward that follows it. Standard film practice is to look at shots in series of threes (Sharff 1982) in order to take into account the influence of the shots preceding and following the shot in question. This practice is particularly important in filming dance, because the choreographed movement must be preserved without interference from interactions between the shots, interactions produced by camera movements and editing.

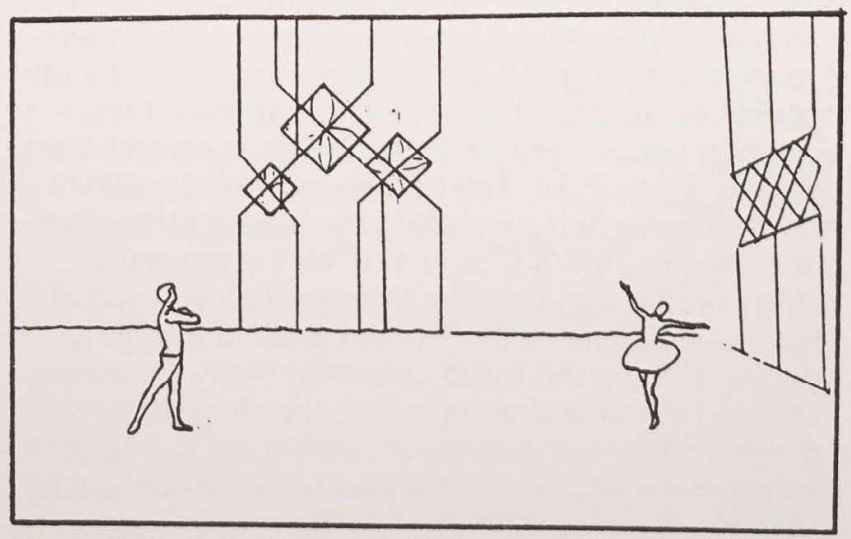

Figures 7a, b, c, d Diamonds (Balanchine, New York City Ballet, Dance in America). From an extreme wide shot when the soloists are farthest apart on the stage (7a), the camera zooms in as the soloists move in toward each other ( $7 \mathrm{~b}$ and c) until the final medium wide shot (7d), when they are closest together on the stage. On the screen they remain just as far apart at the last stage of the zoom (where height

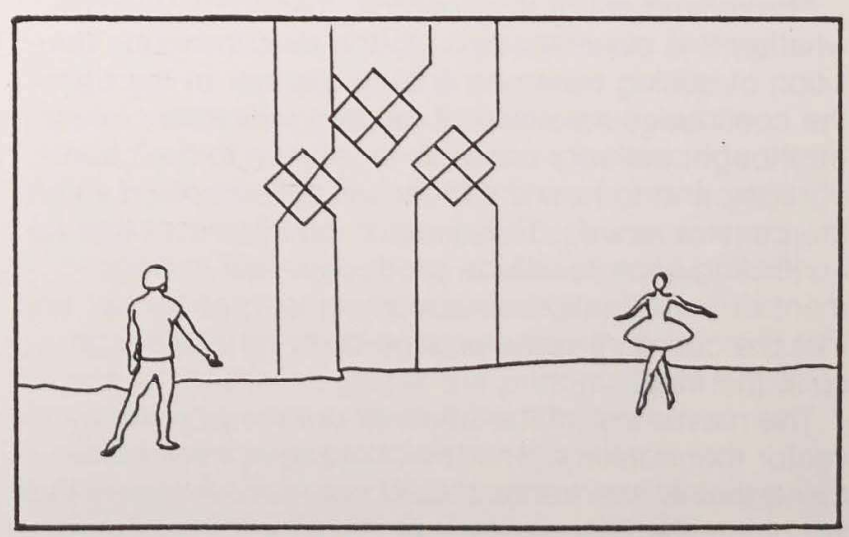

is the limiting factor), but their images are much larger, and the impact of their crossing the final space to meet each other will be much greater in Figure $7 d$ than it would have been had the camera remained as in Figure $7 a$ and had simply allowed their small images to cross and meet. 


\section{Space, Depth, and Movement; the Dancers' Relationship to One Another}

The third question to be considered in the perception of space, depth, and movement is how the dancers are perceived in relation to each other. That, of course, is an essential feature of the choreography that is to be communicated. The angle of view and the focal length of the lens both have important effects on the apparent distance between the dancers, front to back on the stage. Unlike the options available in a studio through the use of cranes and dollys, however, recording live performance in a theater limits the possible combinations of distance and angle from which to shoot. If the director wants to shoot from above, he must place the camera in back of the orchestra or at the first level up in the theater. A telephoto lens would then be used to compensate for the relatively long distance to the stage. If such a camera follows the principal dancer in a full figure long shot, the members of the corps who are posed or making small movement patterns around the edge of the stage will appear to be very close together, front to back (Figure 11b). The principal will appear to be dancing in a narrow space. If the director cuts to a lens of shorter focal length, shooting at a lower angle but closer up, the amount of floor revealed in the frame will still be narrow, but the perceived distance front to back will be enlarged. If an extreme long shot from the top of the house is used, the actual distance will then be shown to be much larger than either of the preceding shots had indicated (Figure 11a). The same telephoto shot from the back of the house will have decreased the value of the movement of the dancers toward the camera: they will appear to move through a very small space. A wider-angle lens will increase the apparent distance through which they will move during the same time. When shot from the top of the house, the corps will appear to be moving up and down on the screen in a flat picture, rather than backward and forward in depth. ${ }^{7}$

When the camera is not moving, one of the best cues for depth comes from the interposition of the dancers as they move around each other and around the other elements in the performing area. When the camera does move, and particularly when a cut is employed from one shot to another, care should be taken to communicate the relative orientations of the dancers. For example, if the camera cuts from the view of a distinctive movement being made by one dancer to another angle on the same dancer finishing the movement, the sequence will preserve the identification of the dancer but it is likely to change the value of the step. The dancer will look the same, but the step will assume a different character when seen from a different point of view. The choice of where in the movement to cut, or whether to cut at all, depends on the dynamics of the steps. Some research has been done on the perceptual "chunking" of movement patterns, that is, on the recognition of the beginnings and endings of movement phrases (Lasher 1979), but knowing where these perceptual divisions occur does not ensure knowing the best
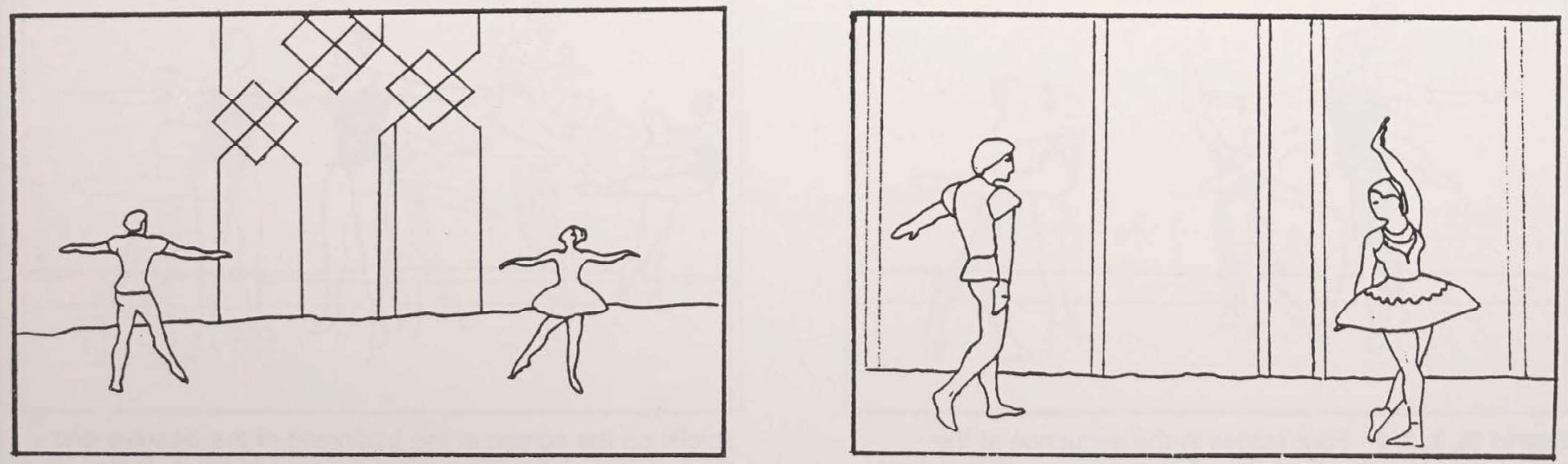

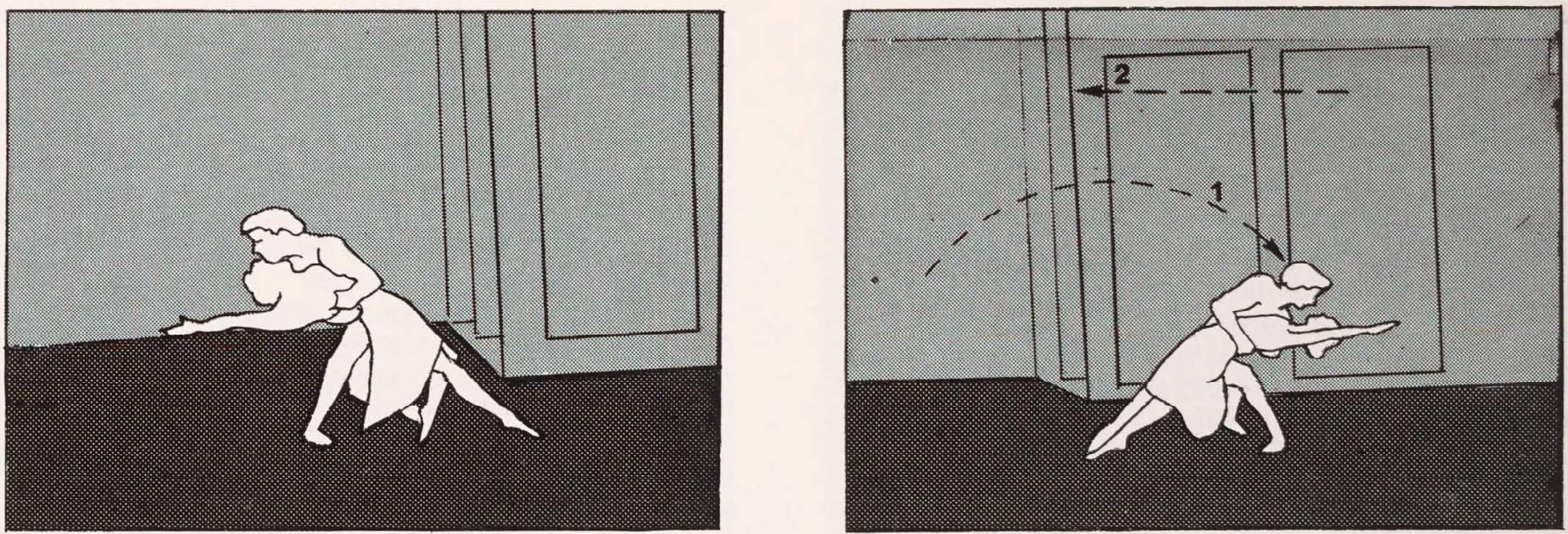

Figures 8a, b Romeo and Juliet (MacMillan, The Royal Ballet, Czinner). The camera swings from left to right as Romeo lifts Juliet from screen left to right (arrow 1). At the same time, the background moves from right to left (arrow 2).

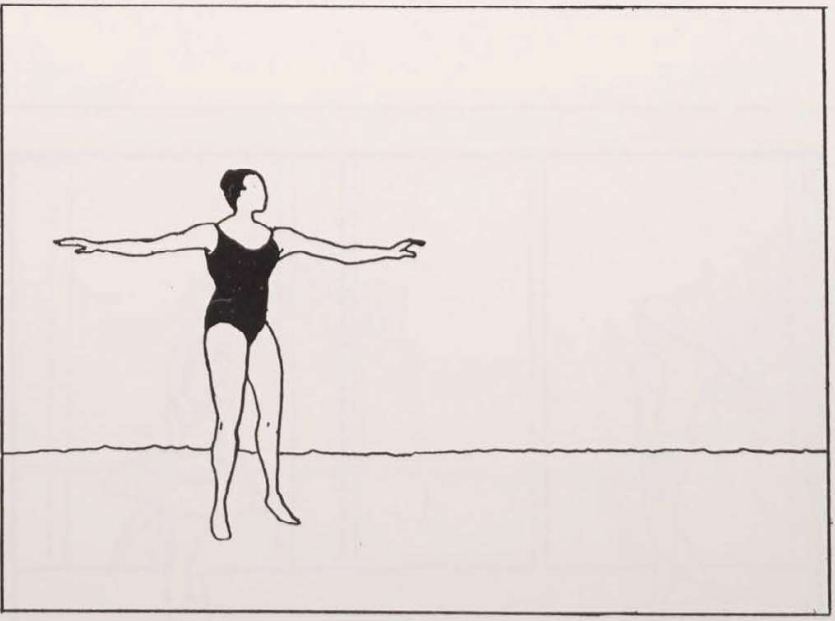

Figures 9a, b, c, d Four stages in the sequence at the beginning of the finale of Four Temperaments (Balanchine, New York City Ballet, Dance in America). (A) The female soloist has traveled screen right away from three couples. When the couples have been cut out of the frame (B), a dissolve begins: $(\mathrm{C})$ the soloist fading out and the three couples fading back in (D). The location of the image of the

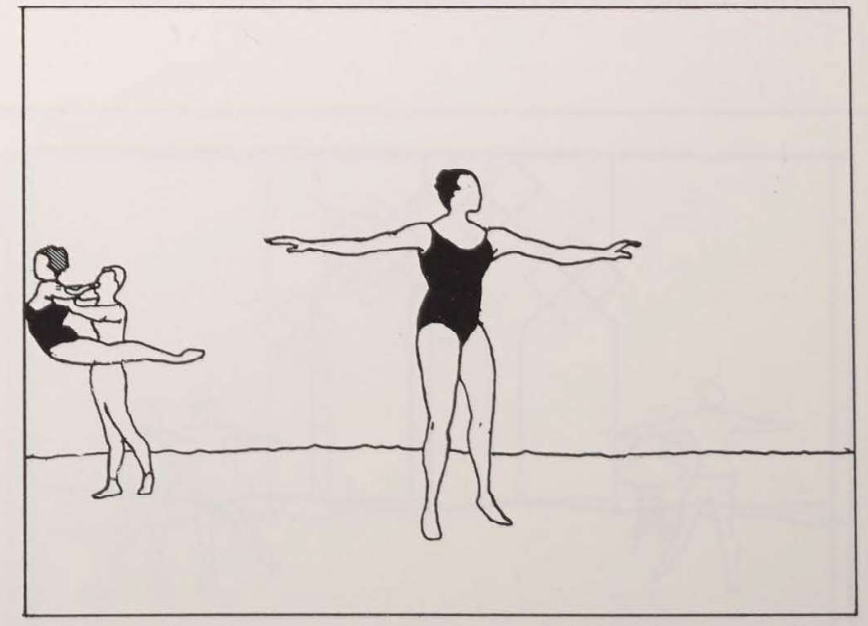

soloist on the screen at the beginning of the dissolve and the fact that all the women are wearing black leotards makes it particularly difficult to keep track of who is where. 
place to cut. The emphasis must surely change if the cut is made in the middle of a phrase, just before the climax of the phrase, or at the end.

There are several ways to keep the viewer aware of the dancers' relative orientation to each other. One of the simplest and most effective is to keep a clear landmark object in view within the shots before and after a cut. An optical effect that can be used to that end, while changing angles, is a matched dissolve: the figure of the principal dancer is in the same spot in both shots and the dissolve simply changes position in terms of direction or distance. This will also preserve orientation of the dancers both to each other and in the whole performance space. Examples of this device used to good advantage may be found in the film of the Alvin Ailey Dance Company, Memories and Visions, and in the previously mentioned "Four Temperaments" ("Choreography by Balanchine, Part l") (Figures 12a, b, c). Dissolves do seem to produce a beat or change in flow of the rhythm just as cuts do, however; it must be made clear by example, early in the film of the dance, that the dissolve is being used only to change angle and not, as in the conventional narrative film sense, to mark the passage of elapsed time. In using this device, moreover, the actual duration of the dissolve must be considered in relation to the movements in the shots being dissolved, so that the full extent of the movement will be seen.

Another optical effect that can be used to communicate the orientations of the dancers relative to each other and to the performance space is a field that is horizontally split into an upper and a lower section. This represents an extreme departure from fidelity or reality, but it may be useful in the case of an intricate pas de deux that takes place in front of a dancing, rather than posed, corps. A medium long shot includ ing the principals, full length in the first shot and full stage in the second shot, will give the detail of the duet along with its relation to the corps. If the principals are dancing alone on the stage but are covering a lot of space, the split field can also be valuable, as it can give the detail in one shot and the direction of travel and the dancers' location on the stage in the other (Figure 13).
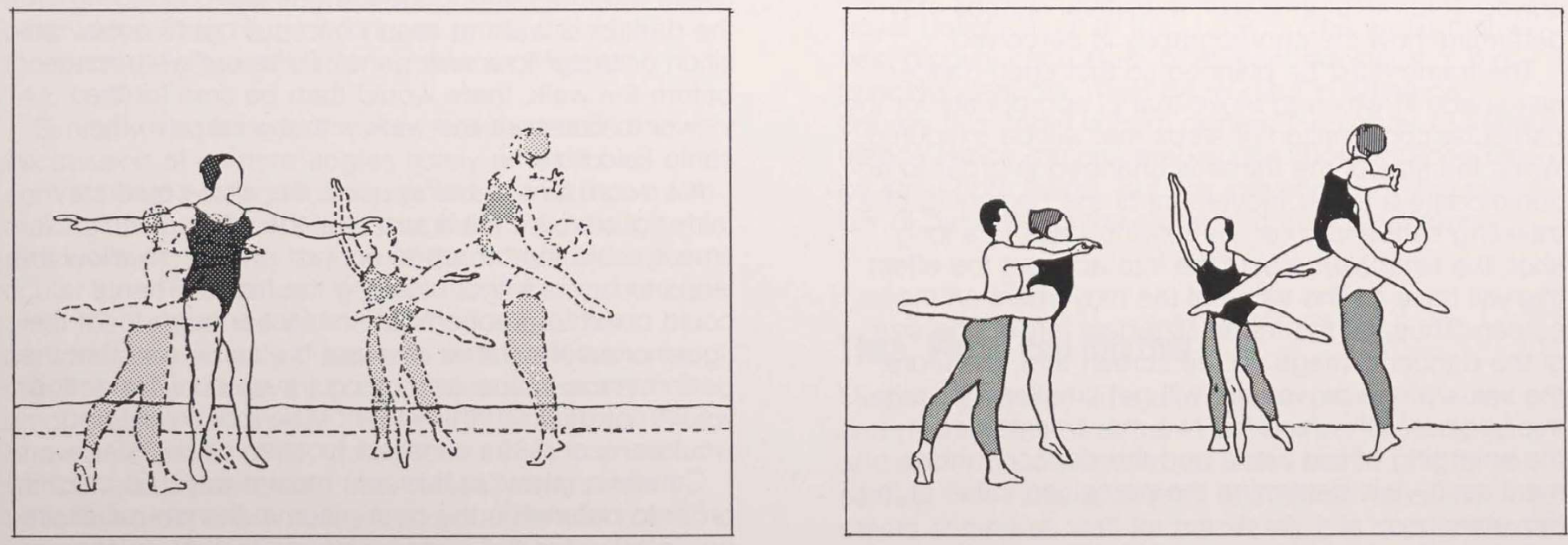
Figures 10a, $b$ Sequence of two shots from the beginning of the men's, variation before the finale of Allegro Brillante (Balanchine, New York City Ballet, Dance in America). Apparent movement backward on the screen (heavy arrows in Figure 10b) in second shot detracts from the continuing forward movement in space that follows.
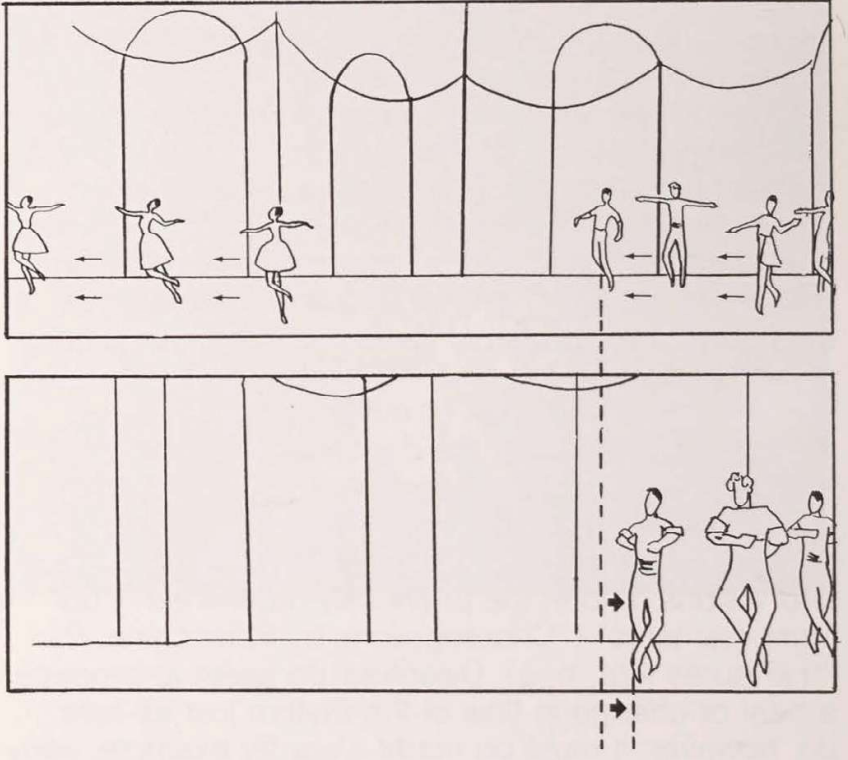

\section{Space, Depth, and Movement in the Dancers' Individual Space}

How the dancers' stances and movements are perceived in terms of their own individual spaces may determine how the choreography is perceived.

The frame must be planned so that each dancer has space in which to move that is appropriate for the particular combination of steps that will be executed. When the size of the frame is changed in order to accommodate a larger movement or the beginning of a traveling sequence, i.e., by moving back to a long shot, the filmmaker must take into account the effect this will have on the value of the movement on the screen. Thus, as the frame size gets larger, the size of the dancer's image on the screen and, therefore, the value of the movement will get smaller. The relative speeds with which the two movements occurthe enlarging of the frame and the dancer's movement itself-will determine the perceived value of the movement.

If the frame is suddenly enlarged through the use of a cut or rapid zoom out, the ensuing leaps or turns - the human effort-will appear insignificant relative to the mechanical or optical movement. If the field enlargement is to be made by cutting to a long shot, it should be made enough in advance of the large movement that the latter will seem larger than those movements that immediately precede it. In other words, if a dancer executes some turns, walks around, and then performs a series of leaps on the diagonal, the camera could stay in tight while the dancer turns in place, but it should use the time between the turns and the leaps (the time during which the dancer is walking around) to pull out to a new position or to cut to a wider shot. If the cut were made before the walk, there would then be time for the viewer to compare the walk with the leaps in the same field size.

If a zoom lens is being used, the shot could stay fairly tight on the full figure until the walk, at which time it could start to open out just enough to allow the leaps to begin without leaving the frame. Then it could continue to open out just fast enough to let the figure cross the frame at about the same rate that the performance space was being traversed. This action would not diminish the value of the movement and would preserve the sense of location on the stage.

Careful analysis in this vein must always be used in order to determine the best place in the overall choreography and in the specific steps at which to change the camera's viewpoint of the dancer. The decision cannot be based solely on the rhythmic needs of the montage.

As far as it is possible to do so, the orientation of a dancer in the performance space as viewed by the theater audience should be maintained in the frame space of the film or television screen, particularly if traversing a lot of space and the pattern of movement 


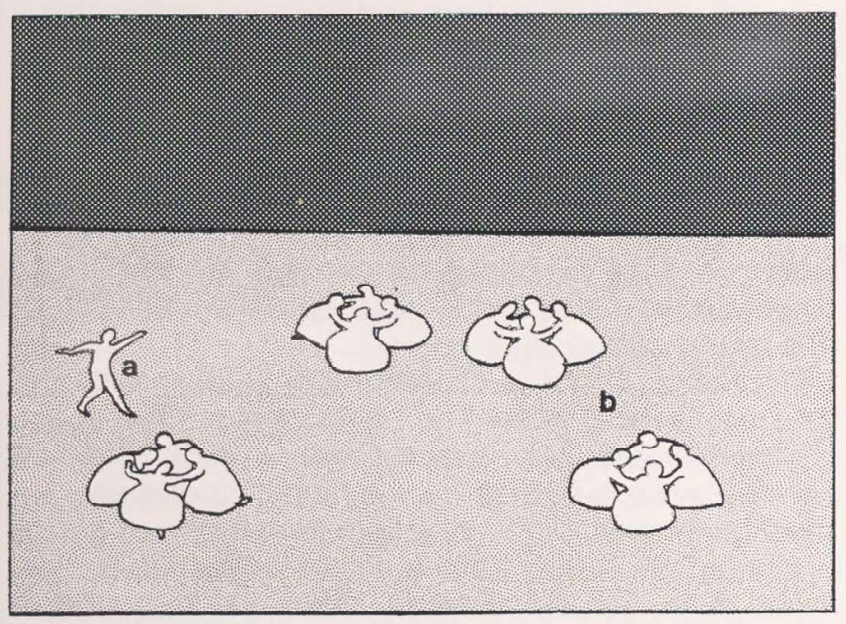

Figure 11a Les Sylphides (Fokine, American Ballet Theater, Live from Lincoln Center). The first shot is wide and taken from a very high angle, using whatever lens was necessary to include the full width of the stage from that distance. The scene appears flat, but the spacing of the groups of dancers on the stage is clear. The soloist is about to move from point A (stage right) to point B (stage left).

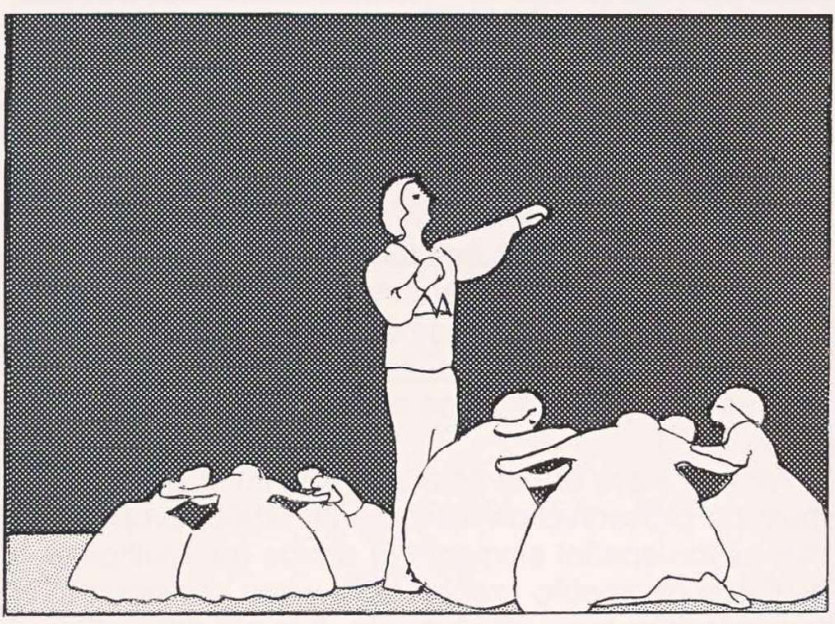

Figure 11b Soloist has arrived at point $B$. The shot is now a telephoto taken from the orchestra level of the theater. The space is compressed, and there is no sense of the distance between the groups of dancers on the stage. are what is important in a particular section of the choreography. Thus, if a dancer is circling the stage in turns, the figure on the screen ideally should go from one side of the frame through the center to the other side of the frame and back to center, while the camera follows the moving dancer (Figures $14 a, b, c)$.

Similarly, the filmmaker is not free to decide the succession of camera angles solely on the basis of conventional editing techniques used in narrative filmmaking. The angle of each shot must be chosen to provide the best way to look at the body for the par ticular steps the dancer is performing. From some angles, the form of the dancer's limbs and of her motions may actually disappear through foreshortening. There has been a strong tendency recently among some perceptual theorists (e.g., Gibson 1979, Shaw and Turvey 1981, Johansson 1982) to assert without proof that motion automatically and directly specifies tridimensional form and that therefore as long as there is any relative motion between dancer and camera, the dancer's location and movements will be communicated correctly. A direct test of those assertions, however, shows them to be unjustified (Amira, Hochberg, and Peterson 1983; Hochberg, Amira, and Peterson 1984). The filmmaker must take into account the momentary two-dimensional image as it is displayed on the screen. A straight shot of a dancer approaching the camera, in a step in which the leg is raised forward, will lose that line completely: the leg will disappear to a point (Figure 15).

Examples can be found in the film of the Bolshoi Ballet's "Giselle" and in "Elite Syncopations" ("The Royal Ballet Salute to the U.S.A."). The angle that will best display each step, or at least the steps of the principal dancers, must be studied in advance. Usually this angle is from or near the center of the audience (since dances are usually choreographed to be seen from there), but it is influenced by the number of dancers on the stage and their placement.

\section{Time, Pace, and Rhythm}

Misperceptions of time and rhythm provide the second major class of errors in perceiving choreography and performance on film. As John Martin (1965) observed, "All these problems of space involve elements of time as well for manifestly it is impossible to move throughout space without occupying time." Once the choices of angle and lens have been made, and once the space and the movement within it have been recorded from those angles and distances, the next decision to be made is how long each shot should remain on the screen. And how will the interaction of the content of the shot with its duration affect the perceived time and rhythm of the dance? 


\section{Lack of Quantitative Research}

No quantitative research has, to my knowledge, been designed to disentangle the effects of content of each shot and the duration of its display on the perception of dance on film. We have available only anecdotal evidence and casual observation on this matter, and these are poor substitutes for research in the case of so precise a factor. Nevertheless, a start is needed, and I will sketch briefly some of what has been written of a theoretical nature, illustrated by such examples as may point to areas of possible experimentation.

What is there about time and rhythm in dance that must be preserved on film? Martin (1965) proposed that all the spatial elements of dance are multiplied, as if in a geometric progression, when considered in terms of their temporal aspects. It is not difficult to perceive such factors as "speed and slowness, gradual accelerations and retardation, or sudden shifts in the rate of movement." It should be possible to portray these elements in a fairly straightforward way on the screen, subject to certain conditions to be discussed shortly. According to Martin,

It is when we approach more complicated involvements in which time and dynamics are concerned together that difficulties appear. This is the category in which the vexed subject of rhythm exists, with its corollaries phrasing and sequential development. ... All rhythms are products of dynamics concerned only incidentally with time. They consist basically of the alternations of accent and unaccent; the time element enters only with the periodicity of the alternations. ... In dance rhythms and phrases, time will play the smallest conscious part, and the spectator will be aware of them chiefly in terms of recurrent spatial patterns with dynamic variations.
Dynamism, indeed, is the heart and soul of rhythm and the vitalizer of the whole art.

This sentiment is echoed by Rudolf Arnheim (1967):

"it is essential for the performance of the dancer

that visual dynamics be clearly distinguished from mere locomotion. ... What counts for artistic performance is the dynamics conveyed to the audience visually; for dynamics alone is responsible for expression and meaning."

The temporal unit of choreography (above that of the step) is the phrase. The components of the dance phrase are isolated by Edwin Denby (1968): "The dance phrase is formed by variation in speed and variation in stress. Its total length is determined by the length of the musical phrase: its total dynamic by the nature of the steps and leaps that are used, by the amplitude that is given them in this particular musical setting." In discussing the relationship of the choreography to the music, Denby points out that the steps often run over the end of the phrase and that accents are distributed sometimes with and sometimes in opposition to the pattern of the music. He notes that the dance phrase may rest "on several accents or climaxes of movement which other movements have led up to or from which they will follow... dance accents frequently do not reproduce the accents of the musical phrases and even when they correspond, their time length is rarely identical with musical time units."

One of Denby's observations that is most important for filming dance is that "the variations of energy in dancing around which a dance phrase is built are what make the dance interesting and alive; and they correspond to a muscular sense, not an auditory one." His observation would seem to be crucial for

Figures 12a, b, c Matched dissolve (Figure 12b) between closeup in Figure $12 \mathrm{a}$ and the wide shot in Figure 12c. The female soloist is in the same position on the screen but the four corps boys have now "entered" from the sides. 
Why Dance Films Do Not Look Right

61

the timing of cuts and camera movements in documints of dance performance. The dance phrase must be noted and preserved, rather than a strict, safe adherence to the musical beat being maintained.

Sometimes, of course, the phrase does correspond with the beat in the choreography itself, but the director and the editor must still be aware of the dancer's emphasis. The dancers' ability to vary the component phrases of the steps - their sense of time values in a sequence of motions - is also, says Denby, a sense of the visual values contained in every sequence:

"The quickening or retarding of motion allows some moments in the movement to be seen more sharply than others, and these stressed moments become the central images around which the observer's mind groups the rest of the motion." Not only the relationships between the music and the choreography itself, but the dancers' phrasing and emphasis within the choreography, must be captured for the film audionce. If a dancer is engaged in extending a measure with a strong balance on point or with a particularly slow pirouette, a cut precisely on the beat would destroy the effect.

In a chapter on rhythm, Ivor Montague (1970) addresses the question of why ballet is so difficult to film:

For us to be conscious of the depth of corps de ballet movements our viewpoint must rise above the level on which they take place. Only then will the tempo of movemont in space be in some degree restored and the composition cease to be a jumble. But too high a viewpoint will distort the figures so that though they will still comprise patterns at approximately correct tempo, they will tend to become dehumanized. ... A greater problem here is the change in viewing detail. ... The music still

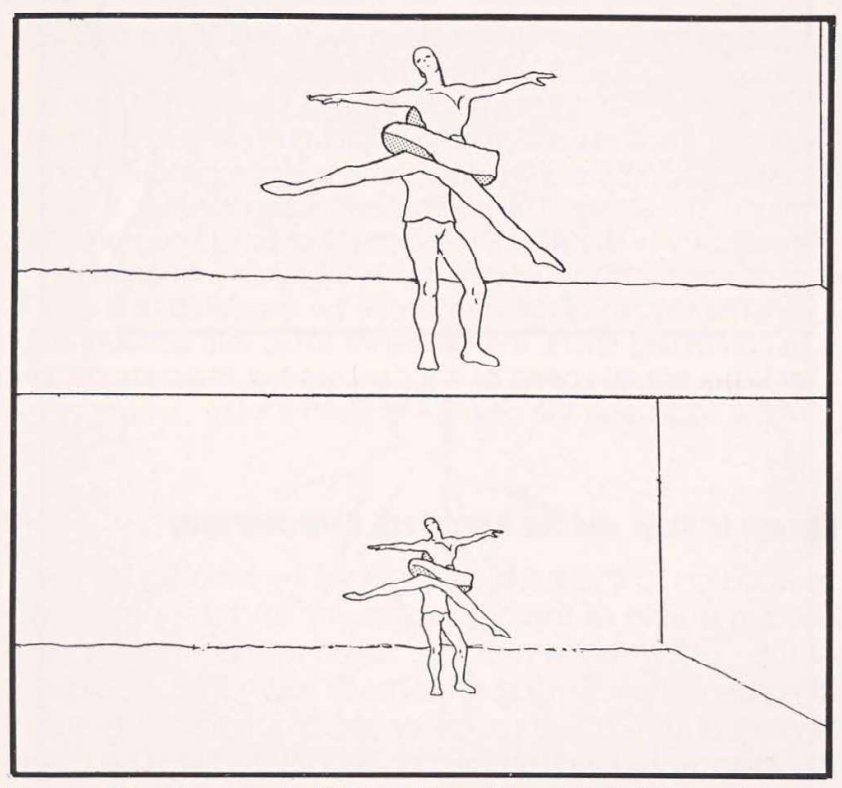

Figure 13 Diamonds (Balanchine, New York City Ballet, record film). Split screen presentation: top view, a close-up showing detail of lift and allowing identification of the dancers; bottom view, a wide shot of the same moment showing the orientation and placement of the dancers within the performing area.
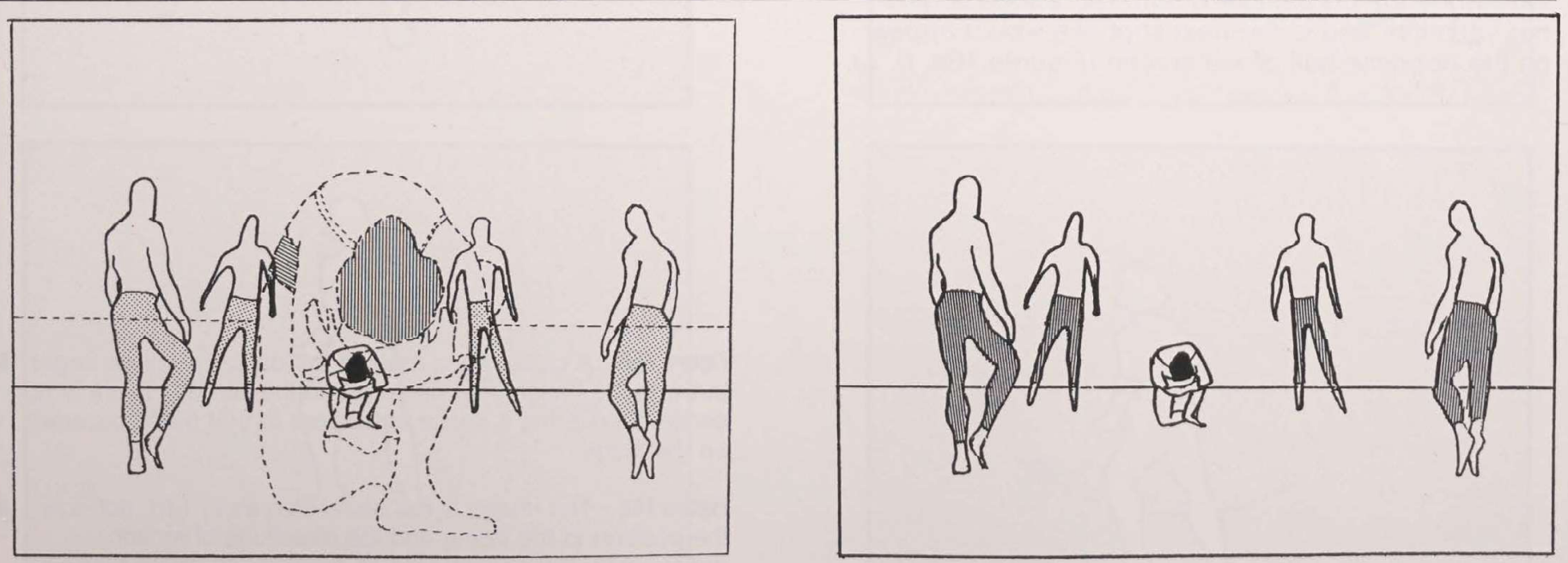
remains the guide. The actual movements of the dancers remain in time. But each cut presents us with a graphic composition so novel compared with the last one that it takes a fractional moment to adjust ourselves to the new composition before we can begin to apprehend its contained movement. The cutting intrinsic to cinema, and necessary to the effective portrayal of any filmed process, here interrupts the rhythm if it is not taken into account.

The rhythm of the dance can be recreated, but only if, in combining shots, the filmmaker takes into account not only the actual speed of the dancers but the composition of the shots and the change of fresh shots.

\section{Change in View and the Perceived Choreography}

In addition to the problems raised by Montague, the pattern or line of the choreography and the phrasing of the performance must be taken into account by the filmmaker. Following a change of view, the absolute rhythm of the music (or other accompaniment to the choreography) will be perceived differently. The magnitude of this effect may be directly related to the degree of change imposed, that is, to the number of compositional elements in the shot that change and have to be reanchored; and it may depend as well on the moment in the choreography when the change is made. A continuum might be set up from least disruptive to most disruptive changes. A matched dissolve between two shots, different only in frame size, that occurs on the musical phrase during a solo might be the least disruptive sort of change in views. A very disruptive change might be produced by a straight cut joining two shots taken from different angles and of different frame sizes. In this case one shot might contain the large ensemble and a soloist in a limbo setting with no landmarks, providing for a massive change in composition; as for timing, the disruptive cut might come during an intricate combination performed by the corps, just before the soloist begins her variation, and in the next shot she would appear on the opposite half of the screen (Figures 16a, b, c)

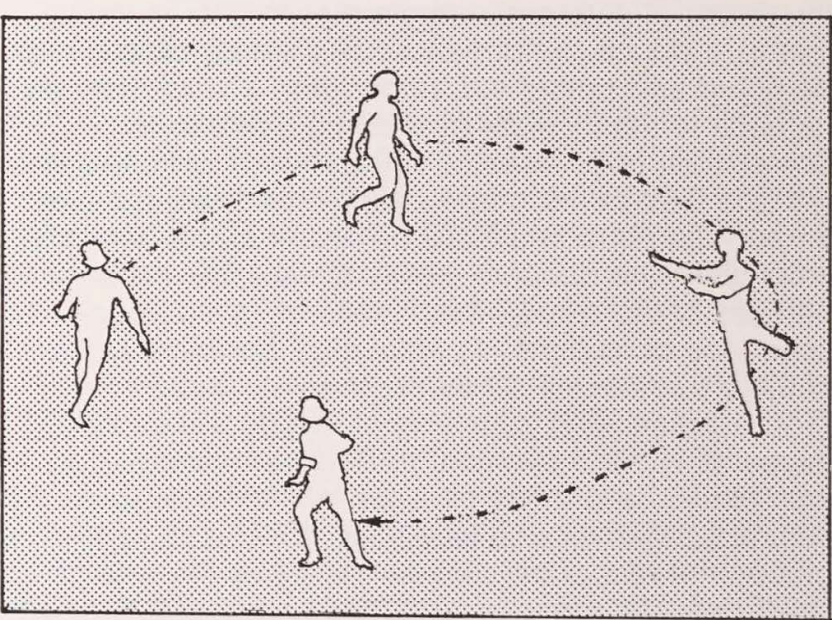

Figure 14a It Starts With a Step (Lotte Goslar Pantomime Circus, The Riverside Dance Festival.) The progression of a dancer in a circle around the stage as it would look in a shot wide enough to include the whole area.
Figure 14b A closer shot, making the dancer's image larger, but with the image kept centered on the screen. There is no sense of covering a space equivalent to that being covered on the stage.

Figure 14c The image is the same size as in 14b, but now the position in the frame and the directions of motion approximate the orientation and progression of the figure on the stage. 

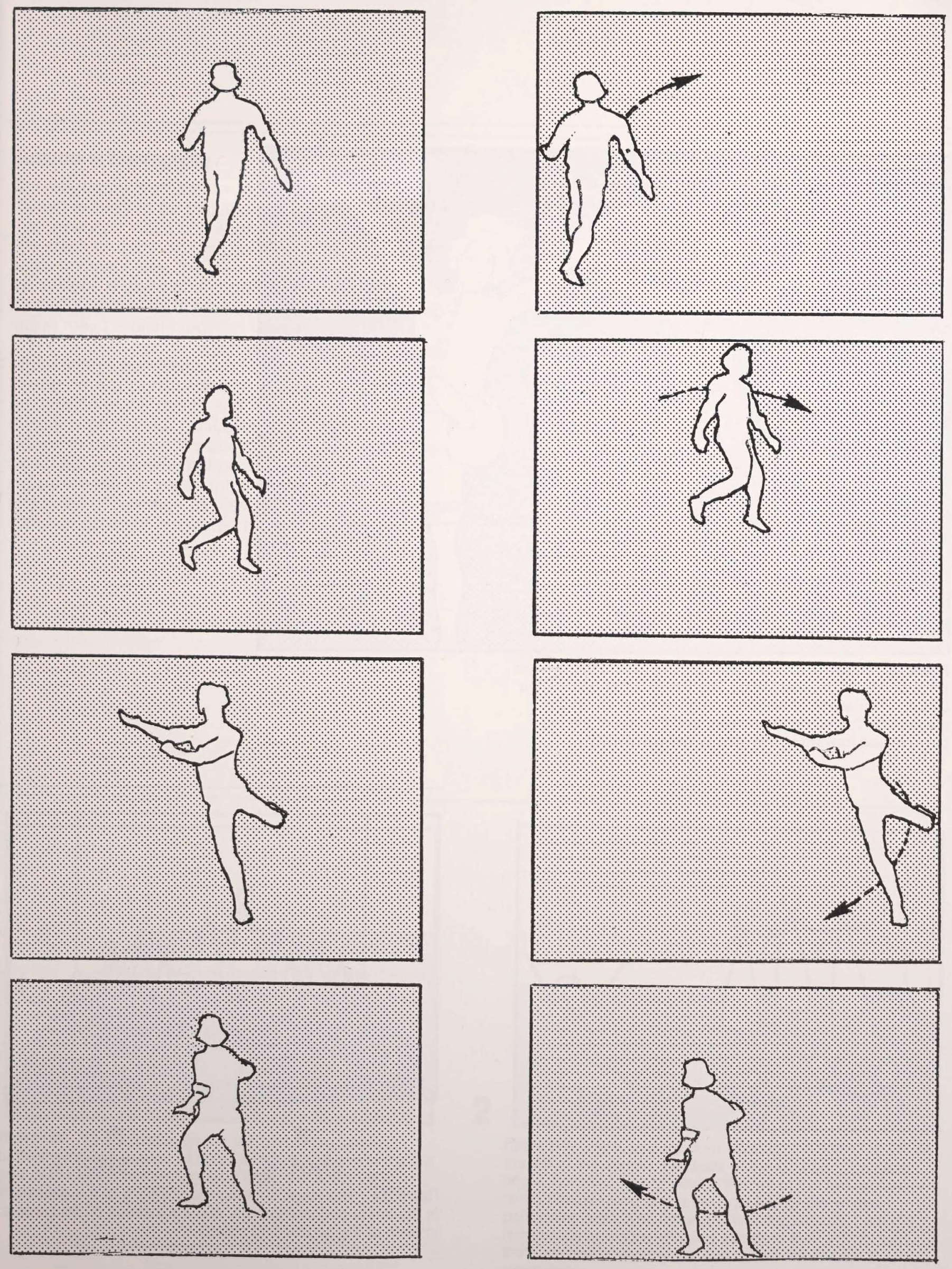


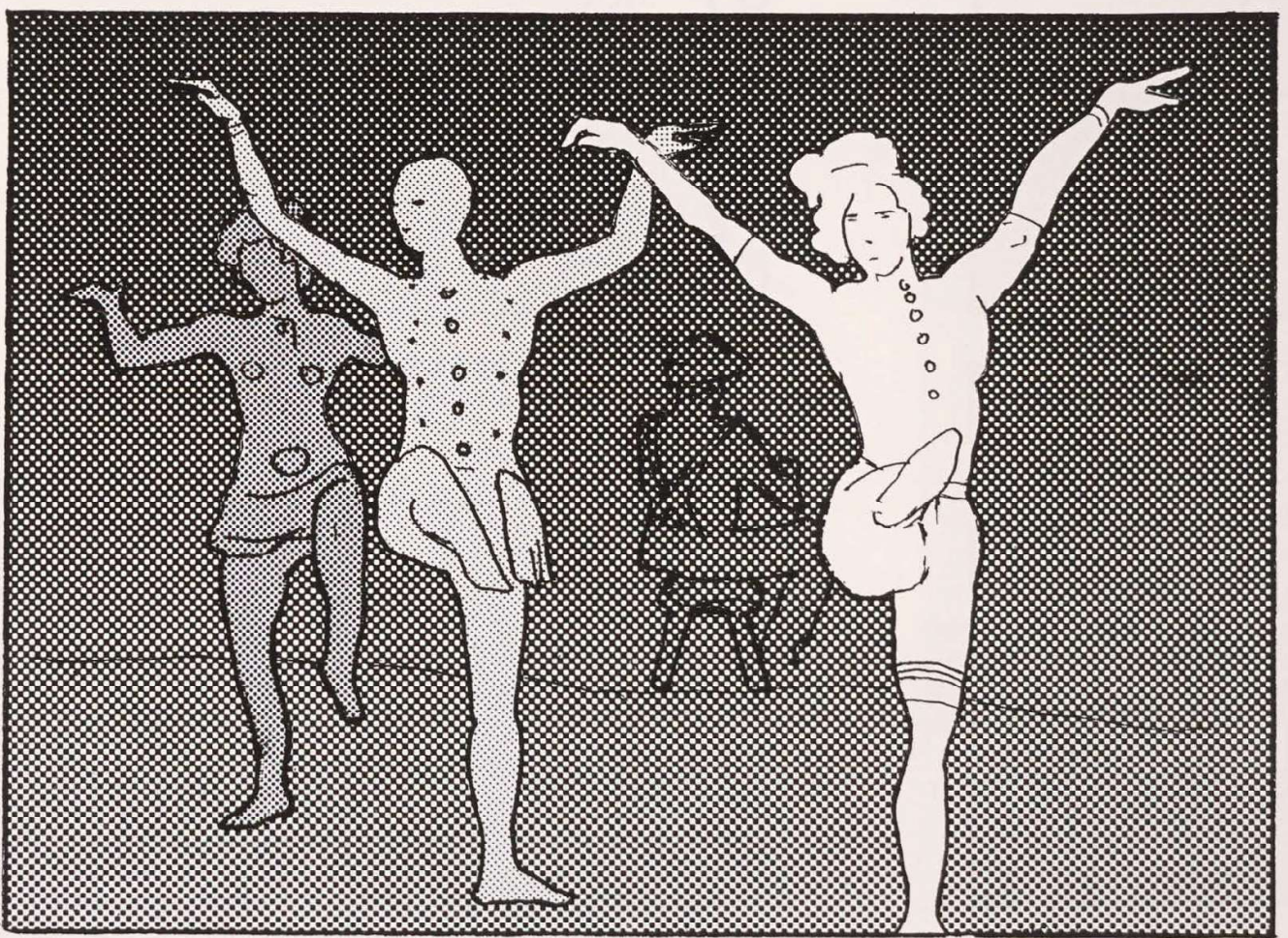

Figure 15 Image of dancers shot coming toward the camera (Elite Syncopation, MacMillan, The Royal Ballet Salutes the U.S.A.). The sense of diagonal progression seen on the stage is dissipated on the screen, and the line of the leg being raised forward in this step is lost completely.

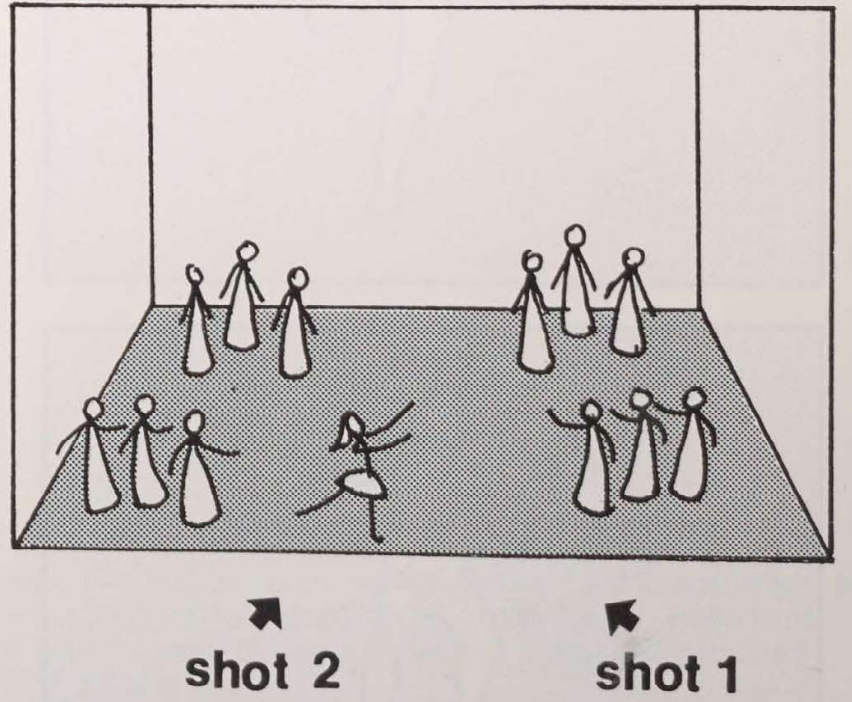

Figure 16a The stage showing the positions of the dancers in the entire performing area, and the location of the camera in each of the succeeding shots. 


\section{Repetition in Choreography}

Choreography is inherently repetitious. Perceived rhythm is likely to be affected by the way in which that repetition in the choreography is filmed. If repetitive steps or combinations are filmed or taped in the same invariant way, the rhythm of the repetition will be evident. If these choreographic similarities are treated in dissimilar filmic ways, the rhythm of the repetition may be disrupted.

The relationships between repetition in the choreography and its complexity and the techniques used in filming have a significant effect on the viewer's comprehension of the dance and, therefore, on how the rhythm is perceived. Controlled experimentation designed to determine the effects of these components would be very useful, not only for application in filming dance but for understanding the more general principles of montage as they must be modified in the communication of structured motions and events.

\section{Summary}

If dance on the screen is to communicate successfully the beauty and excitement that may be generated by dance performed live in a theater, as well as the actual space-time events themselves, the guidelines for how to preserve the impact of that beauty and excitement must be discovered. The principles of visual perception, gained by studying human interaction with the three-dimensional world, should serve two purposes: to specify those aspects of the performance in the theater that define the experience, and to translate to the two-dimensional screen those aspects that must be preserved in order to keep the performance experience intact. If the rules that must govern perceiving dance on the screen are refined and applied in determining the best shots and the best methods of editing to use, perhaps the audience for dance in the movie theater and in front of the television set could be served as well as the audience for sports events on the screen has been

Choreographers and dancers might be presented with as much care and devotion as has been accorded to coaches and baseball players; and the audiences for dance, both live and recorded, might grow apace.

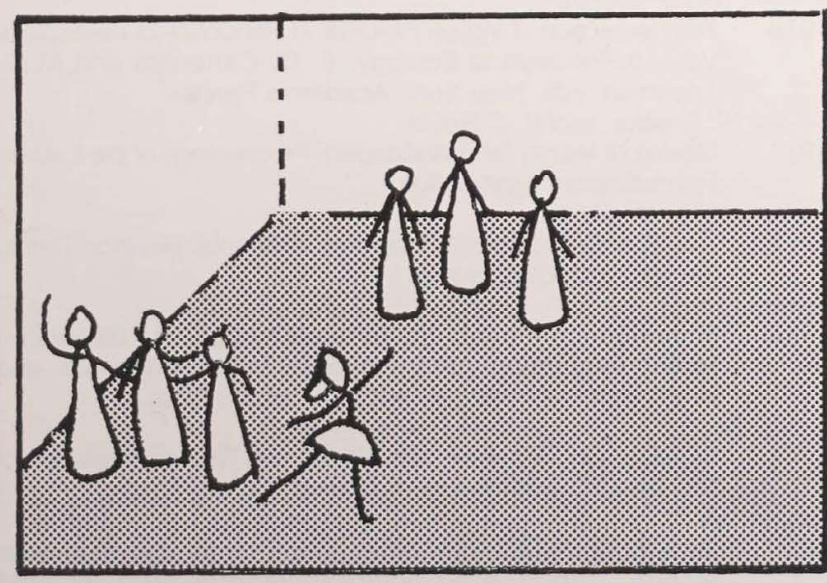

Figure 16b The soloist is on the left part of the screen, between the two groups of corps dancers who are in the left part of the entire performing area (from the audience's viewpoint).

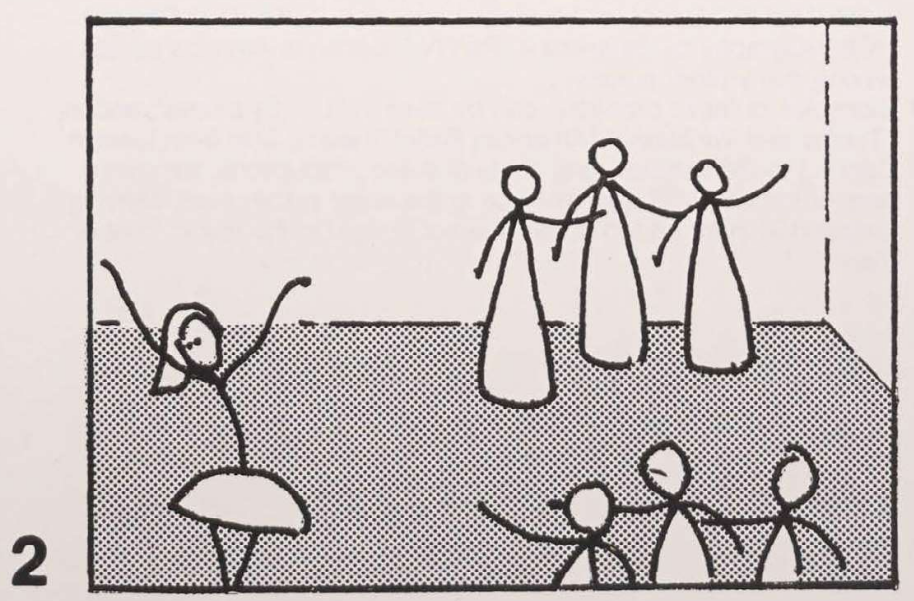

Figure 16c Here, in a medium closeup, the soloist is still on the left of the screen. Because of the change in camera angle, however, she is now to the left of the two groups of corps dancers who are in the right half of the whole performing area. The latter are indistinguishable from the two groups in Figure $16 \mathrm{~b}$ and therefore they will not serve the audience as landmarks that reveal the change in viewpoint. 


\section{Acknowledgment}

This article is based on a chapter from the doctoral dissertation of the author (Brooks 1981). She is grateful to Julian Hochberg for his assistance in the preparation of the figures.

\section{Notes}

1 It may also diminish the value of the moment, as in "Stravinsky Violin Concerto," ("Choreography by Balanchine, Part 1," Dance in America series), in Frederick Ashton's "Monotones II," and in his "Five Brahms Waltzes in the Manner of Isadora Duncan." This is particularly true of the "Live from Covent Garden" version, but is also true in "Trailblazers of Modern Dance," Dance in America series.

2 Examples here include the Tschaikovsky section of "Choreography by Balanchine. Part IV." Dance in America series, and both the Bolshoi Ballet and the Live from Lincoln Center versions of "Giselle.

3 The cues to depth that can be given by stage sets may also affect the perception of movement of the dancers. Such a relationship is particularly evident in Dance in America programs of American Ballet Theatre performing "Billy the Kid" and of the New York City Ballet performing "Tzigane," examples of interactions in which the depth of the dancers' movement is preserved through its relation to the increased sense of depth provided by the forced perspective used in the sets

4 With film, the raw stock may be evenly exposed with a set level of light ("preflashed"), in order to reduce the contrast and bring up some small amount of the detail in the darker sections of the set. This method will not provide as much control over the final results, however, as will actual modification of the lights

5 Examples of this problem can be found in the Live from Lincoln Center series "La Bayadère," in which the magnificent set was lost in shadow, and in the Paul Czinner film Romeo and Juliet, in which the set was often lit so unevenly, particularly when the follow spot was used, that the details of the background appeared and disappeared in a disconcerting manner from shot to shot and as the camera panned following the soloist within a shot

6 This effect can be seen in "Chaconne" " "Choreography by Balanchine, Part III," Dance in America series) and "Ballo de la Regina" ("Choreography by Balanchine, Part IV," Dance in America series), among many other places.

7 Examples of these problems can be seen in "Les Sylphides" and in "Theme and Variations" (American Ballet Theatre, Live from Lincoln Center.) In the extreme long shots of these productions, the members of the corps all appear to be in the same plane, each dancing suspended in air on top of the head of the girl in the row in front of them.

\section{References}

- Amira, L., J. Hochberg, and M. Peterson 1983 Proceedings of the Eastern Psychological Association (abstr.).

- Arnheim, R

1960 Film as Art. Berkeley: University of California Press

1967 Toward a Psychology of Art. Berkeley: University of California Press.

- Brooks, V.

1981 The Art and Craft of Filming Dance as Documentary. Doctoral dissertation, Columbia University. Ann Arbor: University Microfilm Association. 1983

- Brown, J.F

1931 The Visual Perception of Velocity. Psychologische Forschung 14

- Denby, E.

1968 Looking at the Dance. New York: Horizon Press.

- Duncker, K

1938 Induced Motion. In A Source Book of Gestalt Psychology. W. D. Ellis, ed. New York: Harcourt Brace.

- Gibson, J J

1950 The Perception of the Visual World. Boston: Houghton Mifflin.

1979 The Ecological Approach to Visual Perception. Boston: Houghton Mifflin

- Gombrich, E.

1972 Art and Illusion: A Study in the Psychology of Pictorial Representation. Princeton: Princeton University Press.

- Haber, R. N.

1979 Perceiving the Layout of Space in Pictures: A Perspective Theory Based on Leonardo da Vinci. In Perception and Pictorial Representation. C. F. Nodine and D. F. Fisher, eds. New York: Praeger

- Hochberg, J.

forth- The Representation of Space and Events in Video and coming Cinematic Displays. In Handbook of Perception. L. Kaufman and $\mathrm{R}$. Thompson, eds.

L. Amira, and M. Peterson

1984 Extensions of the Schwartz/Sperling Phenomenon: Invariance Under Transformation Fails in the Perception of Objects Moving Pictures. Proceedings of the Eastern Psychological Association (abstr.).

and V. Brooks

1978 The Perception of Motion Pictures. In Handbook of Perception, Vol. 10: Perceptual Ecology. E. C. Carterette and M. P. Friedman, eds. New York: Academic Press

V. Brooks, and P. J. Roule

1977 Movies of Mazes (and Wallpaper). Proceedings of the Eastern Psychological Association.

- $\quad$ and L. Gellman

1977 The Effect of Landmark Features on Mental Rotation Times. Memory and Cognition 5.

- Johansson, G

1982 Visual Space Perception Through Motion. In Tutorials on Motion Perception. A. H. Wertheim, W. A. Wagenaar, and H. W. Liebowitz, eds. New York: Plenum Press.

- Kemp, M

1978 Science, Non-Science, and Nonsense: The Interpretation of Brunelleschi's Perspective. Art History.

- Lasher, M

1979 The Cognitive Representation of an Event Involving Human Motion. Unpublished doctoral dissertation, Columbia University 
- Martin, J.

1965 Introduction to the Dance. New York: Dance Horizons, Inc

- Montague, I.

1970 Rhythm. In The Movies as Medium. L. Jacobs, ed. New York: Farrar, Straus \& Giroux.

- Ornstein, R.

1969 On the Experience of Time. New York: Penguin.

- Richter, J.P.

1970 The Notebooks of Leonardo da Vinci, Vol. 1. New York: Dover

- Sedgwick, H. A.

1980 The Geometry of Spatial Layout in Pictorial Representation.

In The Perception of Pictures, Vol. 1. M. A. Hagen, ed. New York: Academic Press.

- Sharff, S.

1982 Aspects of Cinematic Structures. New York: Columbia University Press.

- Shaw, R., and M. T. Turvey

1981 Coalitions as Models for Ecosystems: A Realist Perspective on Perceptual Organization. In Perceptual Organization. M. Kubovy and J. R. Pomerantz, eds. Hillsdale, N.J.: Lawrence Erlbaum.

- Vorkapich, S

1972 A Fresh Look at the Dynamics of Filmmaking. American Cinematographer, February. 